\title{
Rotor Jackiw-Rebbi Model: A Cold-Atom Approach to Chiral Symmetry Restoration and Charge Confinement
}

\author{
D. González-Cuadra $\odot,{ }^{1,}{ }^{*}$ A. Dauphin $\odot,{ }^{1}$ M. Aidelsburger, ${ }^{2,3}$ M. Lewenstein $\odot,{ }^{1,4}$ and A. Bermudez ${ }^{5}$ \\ ${ }^{1}$ ICFO - Institut de Ciències Fotòniques, The Barcelona Institute of Science and Technology, Av. Carl Friedrich \\ Gauss 3, Castelldefels (Barcelona) 08860, Spain \\ ${ }^{2}$ Fakultät für Physik, Ludwig-Maximilians-Universität, Schellingstr. 4, München D-80799, Germany \\ ${ }^{3}$ Munich Center for Quantum Science and Technology (MCQST), Schellingstr. 4, München D-80799, Germany \\ ${ }^{4}$ ICREA, Lluis Companys, 23, Barcelona 08010, Spain \\ ${ }^{5}$ Departamento de Física Teórica, Universidad Complutense, Madrid 28040, Spain
}

(Received 24 August 2020; accepted 20 November 2020; published 16 December 2020)

\begin{abstract}
Understanding the nature of confinement, as well as its relation with the spontaneous breaking of chiral symmetry, remains one of the long-standing questions in high-energy physics. The difficulty of this task stems from the limitations of current analytical and numerical techniques to address nonperturbative phenomena in non-Abelian gauge theories. In this work, we show how similar phenomena emerge in simpler models, and how these can be further investigated using state-of-the-art cold-atom quantum simulators. More specifically, we introduce the rotor Jackiw-Rebbi model, a $(1+1)$-dimensional quantum field theory where interactions between Dirac fermions are mediated by quantum rotors. Starting from a mixture of ultracold atoms in an optical lattice, we show how this quantum field theory emerges in the longwavelength limit. For a wide and experimentally relevant parameter regime, the Dirac fermions acquire a dynamical mass via the spontaneous breakdown of chiral symmetry. We study the effect of both quantum and thermal fluctuations, and show how they lead to the phenomenon of chiral symmetry restoration. Moreover, we uncover a confinement-deconfinement quantum phase transition, where mesonlike fermions fractionalize into quarklike quasiparticles bound to topological solitons of the rotor field. The proliferation of these solitons at finite chemical potentials again serves to restore the chiral symmetry, yielding a clear analogy with the quark-gluon plasma in quantum chromodynamics, where the restored symmetry coexists with the deconfined fractional charges. Our results indicate how the interplay between these phenomena could be analyzed in more detail in realistic atomic experiments.
\end{abstract}

DOI: 10.1103/PRXQuantum.1.020321

\section{INTRODUCTION}

Quantum field theory (QFT) provides a unifying framework to understand many-body systems at widely different scales. At the highest energies reached so far, the Standard Model of particle physics explains all observed phenomena by means of a relativistic QFT of fermions coupled to scalar and gauge bosons [1]. At lower energies, nonrelativistic QFTs of interacting fermionic and bosonic particles form the core of the Standard Model of condensed-matter physics [2], which explains a wide variety of phases via

\footnotetext{
*daniel.gonzalez@icfo.eu
}

Published by the American Physical Society under the terms of the Creative Commons Attribution 4.0 International license. Further distribution of this work must maintain attribution to the author(s) and the published article's title, journal citation, and DOI.
Landau's seminal contributions of spontaneous symmetry breaking (SSB) [3] and quasiparticle renormalization [4]. In the vicinity of certain SSB phase transitions, the quasiparticles governing the long-wavelength phenomena can be completely different from the original nonrelativistic constituents [5], and even be described by relativistic models analogous to those of particle physics. In fact, it is the careful understanding of this quasiparticle renormalization, which yields the very definition of a relativistic QFT [6-8], and sets the basis for the nonperturbative approach to lattice gauge theories $[9,10]$.

More recently, the range of applications of relativistic theories has been extended to much lower energies, as they also appear in experiments dealing with the coldest type of quantum matter controlled in a laboratory: ultracold neutral atoms [11-16] and trapped atomic ions [17-19]. In contrast to condensed matter and high-energy physics, cold-atom and trapped-ion experiments deal with quantum many-body systems that can be accurately initialized, 
controlled, and measured, even at the single-particle level, turning Feynman's idea of a quantum simulator (QS) $[20,21]$ into a practical reality $[22,23]$. One of the unique properties of cold-atom QSs is the possibility of controlling the effective dimensionality of the model in an experiment. This is particularly important in a QFT context, where interactions tend to be more relevant as the dimensionality is lowered [6], bringing in an increased richness in the form of nonperturbative effects. Moreover, the reduced dimensionality sometimes captures the essence of these nonperturbative effects, characteristic of higher-dimensional non-Abelian gauge theories, in a much simpler arena. Some paradigmatic examples of this trend are the axial anomaly of the Schwinger model [24,25], the strong-weak duality of the Thirring model [26,27], asymptotic freedom and dynamical mass generation in the Gross-Neveu model [28], and the fractionalization of charge by solitons in the Jackiw-Rebbi model [29]. Therefore, the first QSs of QFTs are targeting models in low dimensions [30-35].

We note that the flexibility of these platforms offers an exceptional alternative: rather than using the QS to target a QFT already studied in the realm of high-energy physics or condensed matter, one can design the QS to realise new QFTs which, although inspired by phenomena first considered in these disciplines, lead to partially uncharted territory and give an alternative take on longstanding open problems in these fields. For instance, despite the huge success of the Standard Model of particle physics, the absence of fractionally charged quarks from the spectrum still presents unsolved questions in quantum chromodynamics (QCD), such as understanding the specific microscopic mechanism for the confinement of quarks into mesons and hadrons with integer electric charges [36]. One related problem that remains open is the nature of the confinement-deconfinement transitions at finite temperatures and densities [37] that lead to phases with isolated quarks and gluons - as well as its relation with the restoration of chiral symmetry [38]. Unfortunately, gauge theories in $(1+1)$ dimensions are all confining regardless of the Abelian or non-Abelian nature of the gauge group. In higher dimensions, deconfinement is usually driven by four-body plaquette interactions, which are challenging to implement in cold-atom experiments [30,39]. Therefore, rather than looking for QSs of gauge theories, one can exploit the aforementioned flexibility of QSs to design new QFTs where characteristic phenomena of higher-dimensional non-Abelian gauge theories emerge in strongly correlated phases. In this article, we follow this route, and identify a simple lattice model in $(1+1)$ dimensions that regularizes a relativistic QFT, where the interplay between dynamical mass generation and charge fractionalization leads to confinement-deconfinement transitions of quarklike quasiparticles, the mechanism of which can be neatly understood at the microscopic level.
Although various mechanisms of confinement in QSs have been discussed in the literature [40-55], to the best of our knowledge, our work identifies for the first time a confinement-deconfinement transition between fractionally charged quasiparticles. Moreover, the feasibility of our QS proposal with state-of-the-art cold-atom experiments indicates the possibility of experimentally observing this transition, together with other QCD-like phenomena, such as chiral symmetry restoration at finite densities.

The rest of the paper is organized as follows. In Sec. II, we introduce the Jackiw-Rebbi model and summarize our main results. Starting from a discretized spin-fermion Hamiltonian on a one-dimensional lattice, we show how the continuum quantum field theory emerges in the longwavelength limit. Moreover, we propose a quantumsimulation scheme of this theory using a mixture of ultracold atoms in an optical lattice. In Sec. III, we study the spontaneous breaking of chiral symmetry in the vacuum of the theory, and the corresponding generation of a dynamical mass for the fermionic fields. First, we derive analytically the phase diagram of the continuum model in the large-spin limit. We then extend these results numerically to the lattice, including the effect of quantum and thermal fluctuations, and show how the latter can restore chiral symmetry. In Sec. IV, we analyze the different regimes of quasiparticles that appear on top of the symmetry-broken vacuum. In particular, we calculate the static potential between them, finding how fermionic quasiparticles with fractional charge modify their interactions from repulsive to attractive as we vary one microscopic parameter. The latter corresponds to a deconfinement-confinement transition, as we confirm in the case of nonzero chemical potentials in the thermodynamic limit. Finally, we show how, in the deconfined phase, chiral symmetry is restored even at zero temperature. We present our conclusions and outlook in Sec. V.

\section{THE ROTOR JACKIW-REBBI MODEL}

In this section, we construct the Jackiw-Rebbi model by first introducing a discretized lattice model, where movable fermions are coupled to spins located on the lattice sites. In Sec. II A, we describe the main elements of the corresponding Hamiltonian and we compare it to other models used in high-energy physics such as lattice gauge theories. We also summarize the phase diagram of the model, both in the vacuum as well as for finite densities. In Sec. II B, we present a detailed quantum-simulation scheme for the spinfermion model using a Bose-Fermi mixture of ultracold atoms on a single optical lattice, and show how its whole parameter space is experimentally accessible. Finally, in Sec. II C, we take the continuum limit of the lattice model in the long-wavelength regime, establishing a precise connection between the atomic system and the Jackiw-Rebbi quantum field theory. 


\section{A. Lattice discretization}

To motivate the nature of our model, we note that imposing nonlinear constraints in QFTs is also a source of nonperturbative phenomena that resemble the phenomenology of non-Abelian gauge theories. The $O(N)$ nonlinear sigma model, where a vector field is constrained to take values on the $(N-1)$ sphere, also displays asymptotic freedom and dynamical mass generation [10,56], although the latter cannot be accompanied by SSB in $(1+1)$ dimensions [57]. Remarkably, the $O(3)$ nonlinear sigma model with an additional topological term arises as the long-wavelength description of Heisenberg antiferromagnetic spin chains [58,59]. For leading antiferromagnetic correlations, these systems are effectively described by quantum rotor models, which consist of particles whose internal dynamics are described by rotations on a sphere, such that their angular momentum competes with the interactions that favor a collective orientation [58-60]. This motivates our study of constrained QFTs involving rotors as mediators of interactions between Dirac fermions.

Let us introduce a $(1+1)$-dimensional lattice model that can display SSB of a chiral symmetry, leading to dynamical mass generation, which allows, as we see, the emergence of confinement. We consider a Hamiltonian lattice field theory of fermions $c_{i}, c_{i}^{\dagger}$ and spins $\mathbf{S}_{i}$, both residing at the sites $x_{i}=i a$ of a one-dimensional (1D) chain of $N_{s}$ sites and length $L=N_{s} a$ (see Fig. 1). The fermions hop between neighboring sites with tunneling strength $t$ across a potential landscape set by a spin-fermion coupling $\mathbf{g}=g \mathbf{e}_{z}$, and determined by the spin along the quantization axis

$$
H=\sum_{i}\left[-t\left(c_{i}^{\dagger} c_{i+1}+c_{i+1}^{\dagger} c_{i}\right)+\mathbf{g} \cdot \mathbf{S}_{i} c_{i}^{\dagger} c_{i}-\mathbf{h} \cdot \mathbf{S}_{i}\right] .
$$

Additionally, the spins precess under an external field with both longitudinal and transverse components $\mathbf{h}=h_{\ell} \mathbf{e}_{z}+$ $h_{t} \mathbf{e}_{x}$, the latter controlling the quantum fluctuations of the spins. Although Eq. (1) bares a certain resemblance to the Kondo model of fermions coupled to magnetic impurities $[10,61]$, the fermions are spinless in the present case, and there is no continuous $S U(2)$ symmetry in the coupling.

Let us briefly summarize our findings. Dispensing with the continuous $O(3)$ symmetry of the nonlinear sigma model $[58,59]$, we show that long-range antiferromagnetic order can take place even in $(1+1)$ dimensions, as it is the result of the breakdown of a discrete chiral symmetry [Fig. 2(a)]. These properties can be neatly understood in the long-wavelength limit, where we find an effective Jackiw-Rebbi-type QFT with rotor fields playing the role of the self-interacting scalar field: a rotor Jackiw-Rebbi model. Interestingly, in contrast to the standard JackiwRebbi model [29], the SSB does not take place at the classical level, but requires genuine quantum effects that lead

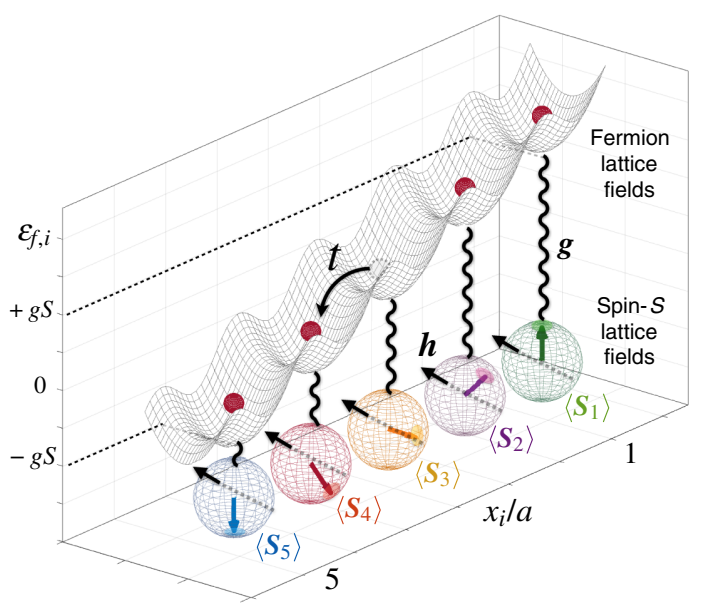

FIG. 1. Discretized rotor Jackiw-Rebbi model: fermions tunnel with strength $t$ against an energy landscape set by the lattice spins $\epsilon_{f, i}=\mathbf{g} \cdot\left\langle\mathbf{S}_{i}\right\rangle$, which additionally precess under a magnetic field $\mathbf{h}$. In the figure, we show a possible configuration of the spins that lead to an energy landscape with a simple gradient. In the text, we show that other configurations that break translational symmetry appear directly in the equilibrium states of the model.

to the nonperturbative dynamical generation of a fermion mass. We also explore how different rotor profiles can arise in the ground state by varying the fermion density, which either leads to fractionally charged or fermion-bag quasiparticles, and prove their stability even in the ultimate quantum limit of spin $S=1 / 2$. In fact, we show that the fermion-bag quasiparticles can be understood as confined pairs of fractional charges, resembling the confinement of fractionally charged quarks in mesons that occurs in the Standard Model of particle physics [Fig. 2(b)]. Interestingly, we find that a confinement-deconfinement transition can be controlled by tuning a single microscopic parameter, and that this quantum phase transition is associated to the restoration of chiral symmetry by soliton proliferation.

Similarly to lattice gauge theories, in our lattice model, Eq. (1), fermion-fermion interactions are mediated by bosonic fields. However, contrary to the former, our model does not possess gauge invariance, a challenging feature to simulate with atomic resources [30]. We show how this simplification allows one to implement the lattice model using state-of-the-art cold-atom QSs in a large regime of realistic experimental parameters. Our results thus show how nonperturbative high-energy phenomena, such as charge confinement, could be investigated using minimal experimental resources. It is important to note that a full quantum simulation of these phenomena in theories such as QCD will require to incorporate gauge-invariant interaction in longer-term quantum simulators. In particular, all the characteristics of the confinement-deconfinement transition between fractionally charged quasiparticles found in our model are not expected to coincide quantitatively with 


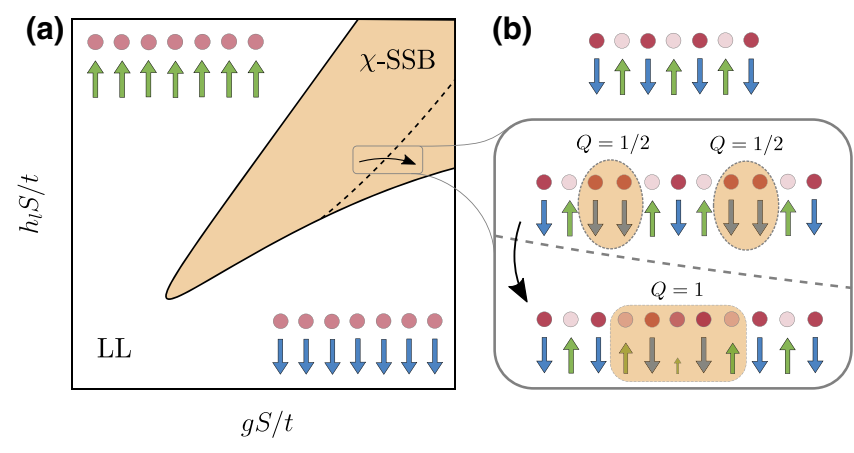

FIG. 2. Phase diagram of the rotor Jackiw-Rebbi model: (a) in the figure, we represent qualitatively the different phases that appear in the half-filled vacuum in terms of the interaction $g$ and the longitudinal field $h_{\ell}$, for a fixed value of the transverse field $h_{t}$ and temperature $T$. Chiral symmetry is spontaneously broken in the shaded region $(\chi-\mathrm{SSB})$, where the fermions develop a dynamical mass and the spins display Néel long-range order, as depicted in the upper panel of (b). We use circles to represent the fermions, where the shade of the color corresponds to the occupation, from white (empty) to red (fully occupied). This region is surrounded by a chiral symmetric phase with a longitudinal paramagnet for the spins, such that the interacting massless fermions form a Luttinger liquid. (b) Within the ordered region, we find two different quasiparticle regimes, separated in the figure by a dashed line. In the first one, deconfined topological defects in the spins bound repulsive quarklike fermions with fractional charges. In the second one, the quasiparticles attract each other, forming mesonlike fermion bags with integer charge. For a finite doping density, the two regimes are separated by a first-order confinement-deconfinement phase transition, which coincides with a chiral symmetry restoration due to the proliferation of defects.

those of the corresponding transition in QCD. However, by further investigating the former in near-term quantum simulators, we can expect to learn universal features about confinement as a general quantum many-body phenomenon, which could then foster further progress on the specific mechanisms taking place in QCD. Let us then start with the proposed scheme for the cold-atom QS.

\section{B. Cold-atom quantum simulation}

The lattice Hamiltonian, Eq. (1), can be realized with a Bose-Fermi mixture of ultra-cold atoms confined in simple state-independent but species-dependent one-dimensional optical lattices. We focus on the $S=\frac{1}{2}$ case, as it presents fewer requirements and, moreover, all the relevant phenomena already appear in this limit of maximal quantum fluctuations. We discuss here a generic situation where the bosonic atoms populate a single internal state, whereas the fermionic atoms can be in two possible states, here labeled as $\sigma \in\{\uparrow, \downarrow\}$. In Appendix A, we present a detailed account for a particular choice of atomic species: ${ }^{87} \mathrm{Rb}$ atoms in their absolute ground state for the bosons, and ${ }^{40} \mathrm{~K}$ atoms in two Zeeman sublevels of the hyperfine ground-state manifold for the fermions. The bosonic and fermionic species will be individually trapped by a single one-dimensional optical lattice, such that the relative strength can be adjusted by tuning the corresponding lattice wavelength. The particular choice of atoms allows for a much deeper lattice for the fermionic species (see Appendix A), such that the dynamics can be described by a Bose-Fermi Hubbard model [62-64] with mobile bosons and effectively immobile fermions. This is described by the Hamiltonian $H=H_{m}+H_{i}$ depicted in Fig. 3, where the external motional degrees of freedom are governed by the grand-canonical Hamiltonian

$$
\begin{aligned}
H_{m}= & -t \sum_{i}\left(\hat{b}_{i}^{\dagger} \hat{b}_{i+1}+\text { H.c. }\right)+\frac{U}{2} \sum_{i} \hat{b}_{i}^{\dagger} \hat{b}_{i}^{\dagger} \hat{b}_{i} \hat{b}_{i} \\
& -\sum_{i} \mu_{i} \hat{b}_{i}^{\dagger} \hat{b}_{i} \\
& +\sum_{i}\left(U_{\uparrow \downarrow} \hat{f}_{i \uparrow}^{\dagger} \hat{f}_{i \downarrow}^{\dagger} \hat{f}_{i \downarrow} \hat{f}_{i \uparrow}+\sum_{\sigma} U_{b \sigma} \hat{f}_{i \sigma}^{\dagger} \hat{f}_{i \sigma} \hat{b}_{i}^{\dagger} \hat{b}_{i}\right) \\
& -\sum_{i, \sigma} \mu_{i, \sigma} \hat{f}_{i \sigma}^{\dagger} \hat{f}_{i \sigma} .
\end{aligned}
$$

Here, $\mu_{i}\left(\mu_{i, \sigma}\right)$ is the local chemical potential for the bosons (fermions), $U\left(U_{\uparrow \downarrow}\right)$ is the on-site Hubbard interaction due to $s$-wave collisions between a pair of bosons (fermions), and $U_{b \uparrow}, U_{b \downarrow}$ stem from the corresponding fermion-boson scattering. In addition, the Hamiltonian for the internal degrees of freedom of the fermionic atoms reads

$$
H_{i}=\sum_{i}\left(\sum_{\sigma} \frac{\epsilon_{\sigma}}{2} \hat{f}_{i \sigma}^{\dagger} \hat{f}_{i \sigma}+\frac{\Omega_{d}}{2} e^{-i \omega_{d} t} \hat{f}_{i \uparrow}^{\dagger} \hat{f}_{i \downarrow}+\text { H.c. }\right),
$$

which includes the hyperfine-state energies for the fermions $\epsilon_{\uparrow}, \epsilon_{\uparrow}$, whose energy difference can be tuned, e.g., by an external magnetic field, and a local term, e.g., a radio-frequency $\omega_{d}$ that induces oscillations between the two fermionic hyperfine states with the Rabi frequency $\Omega_{d}$ [see Fig. 3(b)].

We consider an average filling of one fermion per lattice site, effectively reducing the fermionic Hamiltonian in a rotating frame to a sum of on-site terms, whose dynamics can be expressed in terms of spin- $\frac{1}{2}$ operators $S_{i}^{z}=$ $\frac{1}{2}\left(\hat{f}_{i \uparrow}^{\dagger} \hat{f}_{i \uparrow}-\hat{f}_{i \downarrow}^{\dagger} \hat{f}_{i \downarrow}\right), S_{i}^{x}=\frac{1}{2}\left(\hat{f}_{i \uparrow}^{\dagger} \hat{f}_{i \downarrow}-\hat{f}_{i \downarrow}^{\dagger} \hat{f}_{i \uparrow}\right)$. As discussed in Appendix A, the strength of the local external driving leads to the transverse field $\mathbf{h}_{t}$, and its detuning from resonance $\Delta_{d}=\left(\epsilon_{f \uparrow}-\epsilon_{f \downarrow}\right)-\omega_{d}$ naturally realizes the longitudinal field $\mathbf{h}_{\ell}$ in a rotating frame

$$
\mathbf{h}_{\ell}=-\Delta_{d} \mathbf{e}_{z}, \quad \mathbf{h}_{t}=-\Omega_{d} \mathbf{e}_{x} .
$$

The key of the present scheme is the possibility of controlling the different Hubbard interactions in Eq. (2) with a 


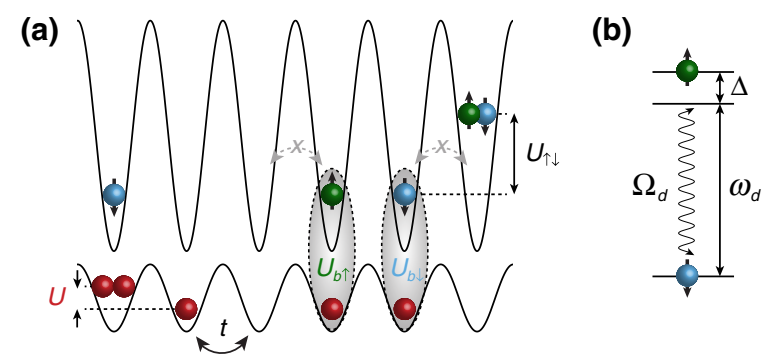

FIG. 3. Bose-Fermi quantum simulator: (a) optical lattice potentials for both the bosonic atoms, here represented by red spheres, and the two-state fermionic atoms, here represented by green (blue) spheres for the $\uparrow(\downarrow)$ states. The bosons have a tunneling amplitude $t$, and the Bose-Hubbard on-site interaction $U$. Conversely, the fermionic tunneling is suppressed by the very deep lattice, and the Fermi-Hubbard on-site interaction strength is $U_{\uparrow \downarrow}$. When residing on the same lattice site, bosons and fermions interact with different strengths $U_{b \uparrow} \neq U_{b \downarrow}$. (b) Driving at frequency $\omega_{d}$ that induces Rabi oscillations between the fermionic states with Rabi frequency $\Omega_{d}$ and detuning $\Delta_{d}$.

single magnetic field using suitable Feshbach resonances. As detailed in Appendix A, the difference between the Bose-Fermi scattering channels readily implements

$$
\mathbf{g}=2\left(U_{b \uparrow}-U_{b \downarrow}\right) \mathbf{e}_{z},
$$

which can be adjusted using a single Feshbach resonance. Finally, in the limit $U \gg t$, the Hamiltonian coincides with the targeted lattice model, Eq. (1), by means of a JordanWigner transformation [65]. In this limit, it becomes clear that we use hardcore bosonic atoms to simulate the fermion fields, and spinfull fermionic atoms to simulate the rotor fields. We note, however, that the relevant physics survives away from this hardcore limit, e.g., we explicitly show a spontaneous breaking of chiral symmetry at a finite value of $U / t$, giving rise to the generation of a dynamical mass for strongly correlated bosons (see Appendix A).

In the Appendix, we also present the detailed calculations of all the relevant parameters for the $\mathrm{Rb}-\mathrm{K}$ mixture, demonstrating a wide range of experimental tunability. We emphasize that the above ingredients have been realized in current cold-atom experiments, and the QS of the model, Eq. (1), is thus realistic. We believe that the main experimental challenge will likely be the preparation of extended regions in the lattice with the proper filling for the bosons and fermions.

In the sections below, we give a detailed account of the results summarized above about the equilibrium states and low-energy quasiparticles of the Hamiltonian, Eq. (1). When discussing these particular phenomena, we comment on specific probing techniques and the temperatures that would be required to observe them. Let us start by describing these effects by means of an effective long-wavelength theory.

\section{Continuum limit}

We now derive the continuum Hamiltonian QFT that captures the low-energy properties of the lattice model, Eq. (1), at half-filling, this is, with fermionic density $\rho=\left(1 / N_{s}\right) \sum_{i}\left\langle c_{i}^{\dagger} c_{i}\right\rangle=1 / 2$, which is considered as the vacuum state in the following. As customary in various $(1+1)$-dimensional systems [66], one starts by linearizing the dispersion relation to obtain a Tomonaga-Luttinger model $[67,68]$. In this case, this amounts to a longwavelength approximation around the Fermi points $\pm k_{F}=$ $\pm \pi / 2 a$, such that $c_{i} e^{-i k_{F} x_{i}} \sqrt{a} \psi_{+}(x)+e^{+i k_{F} x_{i}} \sqrt{a} \psi_{-}(x)$ is expressed in terms of slowly varying right- and leftmoving fermion fields $\psi_{ \pm}(x)$. As detailed in Appendix B, this can be recast in terms of the staggered-fermion discretization of lattice gauge theories [69], where one identifies the Fermi velocity $v_{F}=2 t a$ as the effective speed of light $c=v_{F}$. To proceed with the continuum limit, the spin operators are also expressed in terms of slowly varying fields $\mathbf{S}_{i} \approx \cos \left(k_{N} x_{i}\right) S \mathbf{n}(x)+a \ell(x)$ with $k_{N}=\pi / a$, corresponding to the Néel $\mathbf{n}(x)$ and canting $\ell(x)$ fields $[58,59]$. Performing a gradient expansion and neglecting rapidly oscillating terms yields $H=\int d x \mathcal{H}(x)$ with

$$
\begin{aligned}
\mathcal{H}= & \bar{\Psi}(x)\left[-i c \gamma^{1} \partial_{1}+\mathbf{g}_{s} \cdot \mathbf{n}(x)\right] \Psi(x) \\
& +\left[\mathbf{g} j_{0}(x)-\mathbf{h}\right] \cdot \boldsymbol{\ell}(x),
\end{aligned}
$$

where $\bar{\Psi}(x)=\Psi^{\dagger}(x) \gamma^{0}$ is the adjoint for the spinor $\Psi(x)=\left[\psi_{+}(x), \psi_{-}(x)\right]^{t}$, the gamma matrices are $\gamma^{0}=$ $\sigma^{x}, \gamma^{1}=-i \sigma^{y}$, and the charge density is $j_{0}(x)=$ $\bar{\Psi}(x) \gamma_{0} \Psi(x)$. Additionally, we introduce the coupling $\mathbf{g}_{s}=$ $g S \mathbf{e}_{z}$, and $\partial_{1}=\partial / \partial x$.

This relativistic QFT, Eq. (6), can be understood as a Jackiw-Rebbi-type model [29], in which a Yukawa term couples the fermion-mass bilinear $\bar{\Psi}(x) \Psi(x)$ to the Néel field $\mathbf{n}(x)$, instead of the standard Yukawa coupling to a scalar field $\phi(x)$. Additionally, the rotor dynamics is determined by the precession of the canting field under $\mathbf{g}_{s} j_{0}(x) / S-\mathbf{h}$, instead of the more familiar $\lambda \phi^{4}$ term of the Jackiw-Rebbi QFT. Hence, this precession includes the back action of the matter field onto the mediating fields via the charge density. In the large- $S$ limit, and in phases dominated by Néel correlations, $\mathbf{n}(x)$ and $\boldsymbol{\ell}(x)$ represent, respectively, the orientation and angular momentum of a quantum rotor lying on the unit sphere, such that this QFT, Eq. (6), can be understood as a rotor Jackiw-Rebbi (RJR) model. In contrast to the standard rotor model [60], neighboring rotor fields are not coupled via $O(N)$-symmetric interactions, but rotor-rotor couplings will instead be generated through their coupling to the Dirac fields, and vice versa. As shown below, the lack of a continuous symmetry in Eq. (6) plays a crucial role, and makes the physics of the RJR model very different from these $O(N)$ counterparts. 
If the spins order according to a Néel pattern $\left\langle n_{z}(x)\right\rangle \neq$ 0 , fermions will acquire a mass by the SSB of the discrete chiral symmetry $\Psi(x) \rightarrow \gamma^{5} \Psi(x)$ with $\gamma^{5}=\sigma^{z}$, and $n_{z}(x) \rightarrow-n_{z}(x)$. However, in contrast to the JR model [29], this chiral SSB cannot be predicted classically by looking at the symmetry-broken sectors of the scalar field, i.e., the double-well minima of the classical potential in the $\lambda \phi^{4}$ theory. In our case, the bare rotor term only describes precession of the rotor angular momentum, and does not include a collective coupling of the rotors that would induce Néel order already classically. Instead, in closer similarity to the Gross-Neveu model [28], chiral SSB shall occur by a dynamical mass generation that can only be accounted for by including quantum effects (i.e., rotorfermion loops). On the other hand, there are important differences, as the Gross-Neveu model uses an auxiliary Hubbard-Stratonovich field [70], whereas our rotor fields represent real degrees of freedom with their intrinsic quantum dynamics. As discussed below, this will lead to crucial differences for the chiral SSB, the quasiparticle spectrum, and confinement.

\section{DYNAMICAL MASS GENERATION}

In this section, we study the phenomenon of dynamical mass generation associated to the spontaneous breaking of chiral symmetry in the theory's vacuum. In Sec. III A, we show how this SSB takes place in a region of the quantumfield-theory phase diagram, and uncover the nonperturbative character of this process using a large- $S$ expansion. In Sec. III B, we study the effect of quantum fluctuations, and calculate numerically the phase diagram of the lattice model for various values of $S$. Finally, in Sec. III C, we include thermal fluctuations, and show how the restoration of chiral symmetry shrinks the region in parameter space where the fermion fields have a nonzero mass.

\section{A. Large-S limit}

Using a coherent-state basis [71,72], as discussed in Appendix $\mathrm{C}$, the partition function $Z=\int D[\bar{\Psi}, \Psi$, $\mathbf{n}, \ell] e^{-S_{E}}$ can be expressed in terms of an action $S_{E}=$ $\int d^{2 x} \mathcal{L}(\mathbf{x})$ with the Lagrangian

$$
\mathcal{L}=\bar{\Psi}(\mathbf{x})\left[\hat{\gamma}^{\mu} \partial_{\mu}+\mathbf{g}_{s} \cdot \mathbf{n}(\mathbf{x})\right] \Psi(\mathbf{x})+\left[\mathbf{g} j_{0}(\mathbf{x})-\mathbf{h}\right] \cdot \ell(\mathbf{x}) .
$$

Here, $\mathbf{x}$ is a two-dimensional Euclidean space with imaginary time $x^{0}=c \tau=c(i t)$, and $x^{1}=x$, and the Euclidean gamma matrices are $\hat{\gamma}^{0}=\gamma^{0}, \hat{\gamma}^{1}=-i \gamma^{1}$. The Dirac and adjoint spinors are Grassmann-valued fields $\Psi(\mathbf{x})=$ $\left[\psi_{+}(\tau, x), \psi_{-}(\tau, x)\right]^{t}, \bar{\Psi}(\mathbf{x})=\left[\psi_{-}(\tau, x), \psi_{+}(\tau, x)\right]$, and the charge density is the Grassmann bilinear $j_{0}(\mathbf{x})=$ $\bar{\Psi}(\tau, x) \hat{\gamma_{0}} \Psi(\tau, x)$. Likewise, the spins lead to constrained vector fields which, in the large- $S$ and Néel-dominated limits, correspond to the position $\mathbf{n}(\mathbf{x})$ and angular momentum $\ell(\mathbf{x})$ of a collection of rotors with

$$
\mathbf{n}(\mathbf{x}) \cdot \mathbf{n}(\mathbf{x})=1, \quad \mathbf{n}(\mathbf{x}) \cdot \ell(\mathbf{x})=0 .
$$

Since the Lagrangian, Eq. (7), is quadratic in Grassmann fields, the fermions can be integrated out to obtain an effective action for the rotor fields $S_{\text {eff }}=$ $-\log \left(\int D[\bar{\Psi}, \Psi] e^{-S_{E}}\right)$. In analogy to the Gross-Neveu model [28], where dynamical mass generation can be derived by assuming a homogeneous auxiliary field, we consider $\mathbf{n}(\mathbf{x})=\mathbf{n}, \boldsymbol{\ell}(\mathbf{x})=\boldsymbol{\ell}, \forall \mathbf{x} \in(0, \beta] \times(0, L]$. As shown in Appendix D, the functional integral leads to

$S_{\mathrm{eff}}=\beta L\left\{\left(\frac{\mathbf{g}}{2}-\mathbf{h}\right) \cdot \boldsymbol{\ell}-\frac{\left(\mathbf{g}_{s} \cdot \mathbf{n}\right)^{2}}{4 \pi c}\left[\log \left(\frac{\Lambda_{c}}{\mathbf{g}_{s} \cdot \mathbf{n}}\right)^{2}+1\right]\right\}$,

where we introduce the UV cutoff $\Lambda_{c}=2 t$ set by the bandwith of the bare fermion dispersion on the lattice, Eq. (1).

Exploiting the analogy to the Gross-Neveu model [28], where one deals with $N$ flavors of Dirac fermions and finds the nonperturbative dynamical mass generation in the $N \rightarrow \infty$ limit, we send $S \rightarrow \infty$ to determine the nonperturbative phase diagram quantitatively. Since phases not governed by Néel correlations can also appear, and we are interested in possible quantum phase transitions thereof, we must relax the first constraint in Eq. (8). By considering the explicit construction of the rotor fields in terms of the spin coherent states, we find the Néel and canting fields

$$
\begin{aligned}
\mathbf{n} & =\frac{1}{2}\left(\sin \theta_{A}-\sin \theta_{B}\right) \mathbf{e}_{z}-\frac{1}{2}\left(\cos \theta_{A}-\cos \theta_{B}\right) \mathbf{e}_{x}, \\
\ell & =\frac{S}{2 a}\left(\sin \theta_{A}+\sin \theta_{B}\right) \mathbf{e}_{z}-\frac{S}{2 a}\left(\cos \theta_{A}+\cos \theta_{B}\right) \mathbf{e}_{x} .
\end{aligned}
$$

The fields are thus parametrized by $\theta_{A}, \theta_{B} \in[0, \pi]$, each of which represents the angle of the spin coherent state associated to the $A$ (odd sites) and $B$ (even sites) sublattice, pointing along the great circle contained in the $x z$ plane (see Fig. 1). One can check that the second constraint in Eq. (8) is readily satisfied $\mathbf{n} \cdot \boldsymbol{\ell}=0$, whereas the first one will only be recovered in Néel-dominated phases $\theta_{A} \approx-\theta_{B}= \pm \pi / 2$, where $\mathbf{n} \cdot \mathbf{n} \approx 1$.

By inspecting the effective action, Eq. (9), one finds that it is proportional to $S_{\text {eff }}=\beta L S V_{\text {eff }}\left(\theta_{A}, \theta_{B}\right)$, such that the large- $S$ limit is obtained through the saddle-point equations $\left.\nabla_{\theta} V_{\text {eff }}\right|_{\theta^{\star}}=\mathbf{0}$. The features of the phase diagram can be understood in two complementary regimes: (a) for $h_{t} / t \gg h_{\ell} / t, g / t$, the saddle-point equations will be solved by $\theta_{A}^{\star}=\theta_{B}^{\star}=\pi$, such that all spins are maximally polarized in the direction of the leading transverse field $\left|g_{t}\right\rangle=$ $\otimes_{i}|S, S\rangle_{x, i}$, where $|S, m\rangle_{\alpha, i}$ is the common eigenstate of 
$\mathbf{S}_{i}^{2}, S_{i}^{\alpha}$ with eigenvalues $S(S+1)$, and $m \in\{-S,-S+$ $1, \ldots, S\}$, respectively. This state can thus be understood as a disordered transverse paramagnet, with all spins aligned along the equator of Fig. 1. Since $\left\langle n_{z}\right\rangle=0$, there is no mass generation, and the chiral symmetry remains intact. Therefore, the fermionic sector will correspond to a metallic Luttinger liquid (LL). (b) For $h_{t} / t \ll h_{\ell} / t, g / t$, we find a competition between two distinct phases. If $h_{\ell}>h_{\ell}^{+}$or $h_{\ell}<h_{\ell}^{-}$, where we introduce

$$
h_{\ell}^{ \pm}=\frac{g}{2} \pm \frac{t}{\pi S}\left(\frac{g S}{2 t}\right)^{2}\left[\log \left(\frac{2 t}{g S}\right)+\frac{1}{2}\right]
$$

the saddle-point equations are solved by $\theta_{A}^{\star}=\theta_{B}^{\star}=\pi / 2$, or $\theta_{A}^{\star}=\theta_{B}^{\star}=-\pi / 2$, respectively. These correspond to disordered longitudinal paramagnets with ground states $\left|g_{\ell P}^{+}\right\rangle=\otimes_{i}|S, S\rangle_{z, i}$, or $\left|g_{\ell P}^{-}\right\rangle=\otimes_{i}|S,-S\rangle_{z, i}$, with all spins pointing towards the north or south poles of Fig. 1, respectively. Once again, since $\left\langle g_{\ell P}^{ \pm}\left|n_{z}\right| g_{\ell P}^{ \pm}\right\rangle=0$, there is no mass generation and the fermions are described by a massless Luttinger liquid. On the contrary, if $h_{\ell}^{-}<h_{\ell}<$ $h_{\ell}^{+}$, the saddle points correspond to $\theta_{A}^{\star}=-\theta_{B}^{\star}= \pm \pi / 2$, which yields two Néel antiferromagnets (AF) $\left|g_{N}^{ \pm}\right\rangle=$ $\otimes_{i \in A}|S, \pm S\rangle_{z, i} \otimes_{i \in B}|S, \mp S\rangle_{z, i}$, in which chiral symmetry is spontaneously broken, yielding $\left\langle g_{N}^{ \pm}\left|n_{z}\right| g_{N}^{ \pm}\right\rangle= \pm 1$, respectively. In both cases, the spins of Fig. 1 alternate between the north and south poles, and the Dirac fermions acquire a mass dynamically, accompanied by a so-called scalar condensate

$$
\langle\bar{\Psi} \Psi\rangle=\left\langle\psi_{+}^{\dagger}(x) \psi_{-}(x)+\psi_{-}^{\dagger}(x) \psi_{+}(x)\right\rangle=\Sigma_{0} .
$$

Since the spinor components $\psi_{ \pm}(x)$ correspond to the long-wavelength excitations around momenta $\pm k_{F}=$ $\pm \pi / 2 a$, the scalar condensate of the continuum QFT leads to a periodic modulation of the lattice density $\left\langle c_{i}^{\dagger} c_{i}\right\rangle=\frac{1}{2}+$ $(-1)^{i} \Sigma_{0} a$. This phase is reminiscent of a charge-density wave insulator in electron-phonon systems. However, in these systems, Peierls' argument shows that the 1D metal is always unstable towards the insulator [73], instead of showing different phases separated by quantum critical lines, as occurs for our rotor Jackiw-Rebbi QFT, Eq. (11). Let us remark once more that, while in the standard JR model [29] chiral SSB can be understood by means of perturbation theory about the classically SSB sectors in $g \ll 1$, the mass is dynamically generated in our case, and cannot be understood perturbatively, which can be appreciated by the fact that the $\log (S t / g)$ dependence in Eq. (11) cannot be Taylor expanded for small $g$.

\section{B. Quantum fluctuations and Ising universality class}

Let us now benchmark the above large- $S$ calculations with numerical results based on a Hartree-Fock (HF)
[74] self-consistent mean-field method, and a matrixproduct-state (MPS) [75,76] formulation of the densitymatrix renormalization group (DMRG) [77]. This serves a twofold purpose: on the one hand, both methods are directly applied to the discretized model on the lattice, Eq. (1), and can thus be used to identify the parameter regime where the continuum QFT predictions, Eq. (9), are recovered. On the other hand, the quasiexact MPS method gives direct access to corrections of the large- $S$ predictions for finite values of $S \in\left\{\frac{3}{2}, 1, \frac{1}{2}\right\}$, testing the dynamical mass generation in the regime of large quantum fluctuations. Likewise, we can adapt the HF method to nonzero temperatures $T$ and chemical potentials $\mu$ in order to explore the role of thermal fluctuations and finite densities (see Appendix E).

Figure 4 contains our results for the zero-temperature half-filling phase diagram as a function of $\left(g S / t, h_{\ell} S / t\right)$ for various values of the transverse field $h_{t} S / t$. In the background, we represent the Néel order parameter, $n_{z}=$ $\left(2 / N_{s}\right) \sum_{x} \mathbf{e}_{z} \cdot \mathbf{n}(x)$, obtained by averaging over the HF estimate of the Néel field $\mathbf{n}(x)=(1 / 2 S)\left(\left\langle\mathbf{S}_{2 i}\right\rangle-\left\langle\mathbf{S}_{2 i-1}\right\rangle\right)$. In Fig. 4(a), one can see how the HF predicts an intermediate region, here depicted in red, displaying antiferromagnetic long-range order $n_{z} \approx 1$ due to the SSB of the discrete chiral symmetry. In order to test the validity of our QFT predictions based on the phenomenon of dynamical mass generation, we benchmark these numerical results against the critical lines of Eq. (11), which are depicted as solid black lines in Fig. 4(a). In analogy to the large$N$ limit of other strongly coupled QFTs [70], we must rescale the coupling to obtain physical results for $S \rightarrow \infty$, such that $g S$ remains finite. From the comparison of the $\mathrm{HF}$ and large- $S$ results, we understand that it is the regime of $g S \ll t$ (i.e., couplings much smaller than the UV cutoff), where we can recover the continuum QFT from the lattice discretization, as typically occurs in asymptotically free lattice field theories.

Note that, in fact, the lattice theory already agrees with the continuum predictions for intermediate couplings $g S \sim 0.25 t$, which is a sensible fraction of the UV cutoff, and signals the wide validity of the aforementioned scheme of dynamical mass generation. It is worth mentioning, however, that the SSB mechanism that yields the Néel phase is valid for an even wider range of parameters. Indeed, around any of the critical lines of Fig. 4(a), there will be an effective continuum QFT, albeit with different renormalized parameters that require us to rely on numerical methods or experimental QSs. This wider parameter regime is useful in light of the coldatom realization presented above, which will be able to probe antiferromagnetic correlations in a regime more favorable than the one set by super-exchange mechanisms [78-83]. In particular, for larger values of $g S$, the order survives to larger temperatures, as we also show below. 

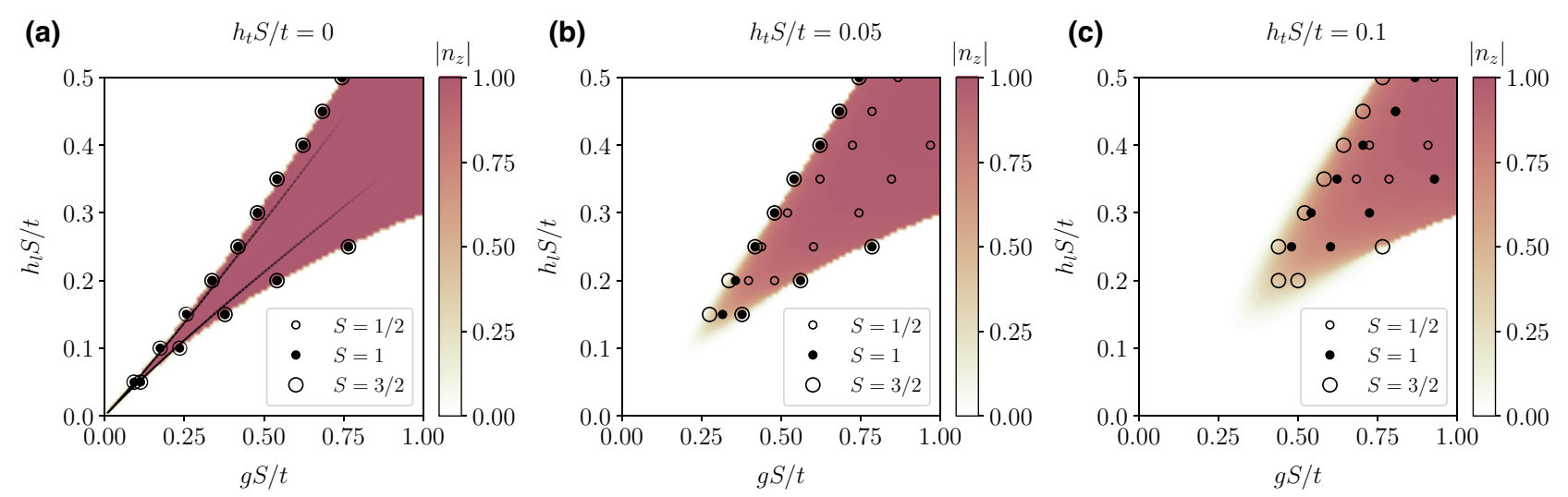

FIG. 4. Dynamical mass generation at finite $S$ : in the background of the different phase diagrams represented in the figure, we show the absolute value of $n_{z}$ calculated with the HF method for a periodic chain of $N_{s}=64$ sites and $N_{f}=32$ fermions. The different dots correspond to the critical points separating the AF from the LL phase, obtained using iDMRG for various values of $S$. (a) When quantum fluctuations are absent, all the points coincide with the HF solution and the large-S predictions for the whole phase diagram. The solid lines correspond to the solution obtained using the large- $S$ expansion in the continuum model, Eq. (11), agreeing with the lattice critical lines for small values of $g S / t$. (b) In the presence of finite quantum fluctuations, the HF solution departs from the correct ground state, although it provides a good approximation even for small values of $S$ in the case of small fluctuations. (c) The AF region shrinks as $h_{t} S / t$ increases, and it does it faster for smaller values of $S$. However, this phase can always be found, even in the limit $S=1 / 2$, provided that $g S / t$ and $h_{\ell} S / t$ are sufficiently large.

The generation of a dynamical mass described in this section can be implemented following the previous coldatom scheme, and experimentally probed using standard detection techniques. In particular, the scalar condensate (i.e., charge-density-wave ordering) can be readily probed by measuring the imbalance $I=\left(n_{A}-n_{B}\right) /\left(n_{A}+\right.$ $n_{B}$ ) between the occupation of the bosonic atoms on $A$ and $B$ sublattices by using superlattices [84-86] or via noise correlations $[16,87,88]$. The antiferromagnetic Néel ordering can be further revealed by evaluating the imbalance observable, or the noise correlations, for the fermionic atoms in a spin-resolved manner, which requires separating the two hyperfine states during the detection by means of a Stern-Gerlach sequence [79], or other similar techniques $[80,89]$. Since in the SSB phase, however, there are two energetically degenerate configurations shifted by one lattice site, and the experiment will consist of many independent copies of the one-dimensional chains, ensemble-averaged observables may fail to signal the phase transition without an additional term that weakly breaks the symmetry between the configurations. If a small symmetry-breaking field cannot be globally introduced in all these copies, one may resort to a combination with quantum gas microscopy $[82,90,91]$, which now also enables full spin and charge readout $[92,93]$.

Let us now explore the effect of finite $S$ and nonzero $h_{t}$. In Fig. 4(a), the circles represent the critical values of the SSB phase transition for different values of $S$, and are obtained with the MPS method based on the iDMRG scheme for an infinite chain [76]. These critical points are estimated by localizing the divergence of the spin susceptibility, $\chi_{S}=\partial n_{z} / \partial(g S / t)$, where we use bond dimension
$D=200$ and a four-site repeating unit cell. As can be observed in the figure, for a vanishing transverse field, the critical points for different $S$ are all arranged along the same critical line, which, furthermore, delimits the Néelordered phase obtained with the HF method, and agrees with the large- $S$ predictions, Eq. (11), in the regime where we expect to recover the continuum QFT from the lattice regularization. We note that, in our model, changing the value of the spin $S$ for a vanishing $h_{t}=0$, does not modify the quantum fluctuations, such that the large- $S$ prediction works equally well for any value of $S$. This contrasts the typical situation in models with $O(3)$ symmetry, where the classical limit is associated with $S \rightarrow \infty$, and quantum fluctuations appear as soon as $S$ is finite. Moreover, as outlined in the Appendix, we confirm that there is no qualitative distinction in the underlying physics for integer or half-integer spins, as occurs for models with a continuous $O(3)$ rotational symmetry.

In Figs. 4(b) and 4(c), we represent the phase diagram as the transverse field is switched on, which introduces quantum fluctuations on the spins. In this limit, we observe how the long-range Néel phase shrinks as a result of the competing quantum fluctuations. We also observe that, as the value of $S$ increases, a better agreement with the HF and QFT predictions is obtained, confirming the generic expectation. Note that this agreement is remarkable, given that the considered values of the spins $S$ are still very far away from the large- $S$ limit.

So far, our numerical benchmark has focused on the SSB captured by the Néel field. Let us note, however, that the dynamical mass generation refers to the fermionic sector, and the gap opening is associated with an underlying 


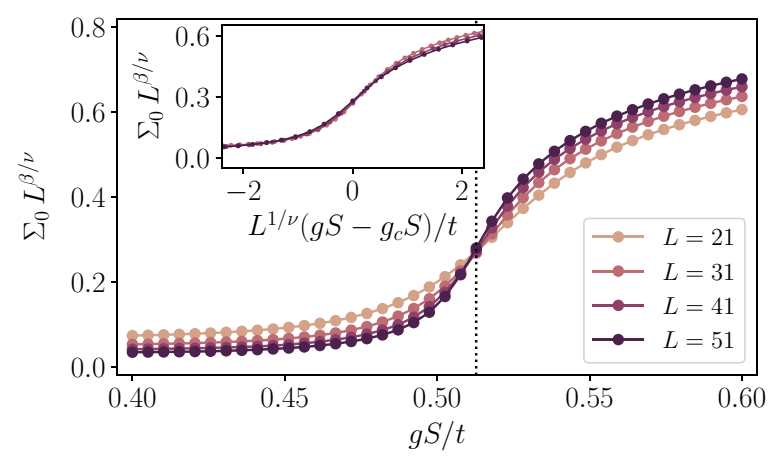

FIG. 5. Scalar condensate and chiral SSB: we represent the scalar fermion condensate $\Sigma_{0}=\langle\bar{\Psi} \Psi\rangle$ in terms of $g S / t$ for the ground state of a half-filled chain of different lengths $N_{s}$, with $h_{\ell} S=0.3 t$ and $h_{t} S=0.05 t$. The results are obtained using DMRG for $S=1 / 2$. Using the critical exponents of the 2D Ising universality class $(v=1, \beta=1 / 8)$, the lines cross at the critical point obtained for the infinite system with iDMRG, and collapse for an appropriate rescaling (inset).

nonzero scalar condensate $\Sigma_{0}=\langle\bar{\Psi} \Psi\rangle$. In order to extract the value of this condensate from the lattice simulations, we use $\Sigma_{0}=\left(1 / N_{s}\right) \sum_{i}(-1)^{i}\left\langle c_{i}^{\dagger} c_{i}\right\rangle$, where the expectation value is calculated with the MPS ground state obtained using a DMRG algorithm with bond dimension $D=200$ for finite chains of variable length $L=N_{s} a$ and unit lattice spacing $a=1$. In Fig. 5, we represent the finite-size scaling for the scalar condensate of the $S=1 / 2$ model. The crossing of the lines in the main panel serve to locate the critical point of the model $g S_{c} / t$, which agrees with our previous iDMRG results based on the Néel order parameter. Hence, this shows that the chiral SSB occurs via the simultaneous onset of a Néel antiferromagnet and a scalar fermion condensate, which corresponds to a charge density wave as seen from the lattice perspective. Moreover, these results allow us to identify the universality class of the corresponding chiral phase transition. As proved by the data collapse shown in the inset of Fig. 5, the critical exponents correspond to those of the $(1+1)$ Ising universality class.

\section{Thermal fluctuations and chiral symmetry restoration}

Let us now move on to the study of the corrections due to thermal fluctuations. In Fig. 6, we represent sections of the phase diagram as a function of $\left(g S / t, h_{\ell} S / t\right)$ for several values of the temperature $T$ obtained with the HF method. One can observe how the area that encloses a dynamically generated mass, characterized by the Néel order parameter $n_{z}$, shrinks with increasing $T$. Therefore, for sufficiently high temperatures, the chiral SSB phase would eventually disappear in favor of the disordered paramagnet and the massless Dirac fermions, both of which respect the chiral symmetry.

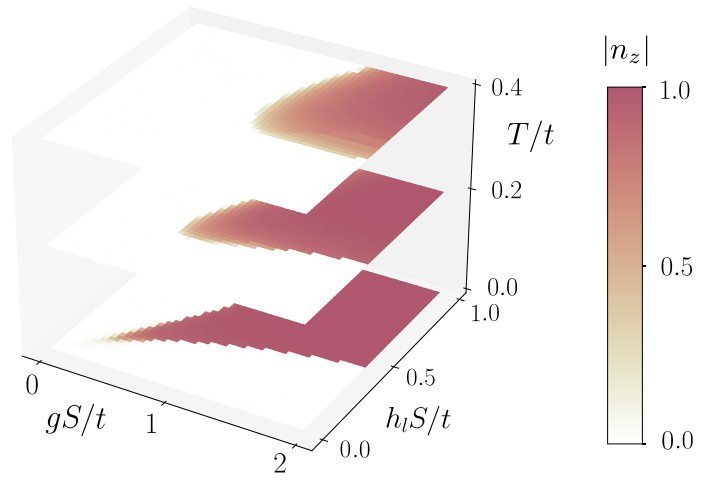

FIG. 6. Chiral symmetry restoration at finite temperatures: phase diagram for different $T / t$ and $h_{t} S=0.05 t$, where we represent the Néel order parameter $n_{z}$ calculated in the HF approximation. The AF phase, where chiral symmetry is spontaneously broken, shrinks as the temperature increases. In the high-energyphysics lore, one says that chiral symmetry is restored at high temperatures.

As shown in Fig. 6, the required temperature scale for a robust observation of the different phases may lie above that of the tunnel coupling $t$, which is a very promising feature of our QS scheme. Using state-of-the-art cooling techniques, temperatures as low as $T / t=0.2$ have been reported for two-component Fermi gases [82] and $T / U=$ 0.05 for bosonic atoms [94]. As displayed in Fig. 6, the dynamical mass generation leading to the antiferromagnetic order and the scalar condensate can be accessed at larger temperatures $T / t \sim 0.4$. The underlying reason is that the onset of antiferromagnetic correlations does not rest on the super-exchange mechanism, the scale of which is $t^{2} / U \ll U$. In our case, the mechanism is the dynamical breaking of chiral symmetry, the scale and gap of which is directly set by the interaction coupling $g \sim U_{b f}$, which can be on the order of the Bose-Fermi interactions $U_{b f}$.

Let us now interpret these results in light of thermal chiral symmetry restoration in the Standard Model. In particular, for a large region of its phase diagram, the vacuum of QCD is expected to break spontaneously a chiral symmetry associated to the quarks' flavor [95]. This mechanism is confirmed experimentally by the measured mass of light baryons, such as protons and neutrons, where chiral symmetry breaking yields the largest contribution to their masses [96], while only a small part comes from the masses of their constituent quarks. On the other hand, at very high temperatures or densities, corresponding to the first instants after the Big Bang or to the dense core of neutron stars, respectively, chiral symmetry is restored and quarks become massless, as shown in experiments involving heavy-ion collisions [97]. This phenomenon is captured by several effective theories of nucleons, such as the Nambu-Jona-Lasinio quantum field theory [98] and, as discussed above, also occurs in our model. 
There are, however, several unsolved questions in QCD regarding the restoration of chiral symmetry. One is whether a phase transition at finite $T_{c}$ or a crossover exists for intermediate values of the baryon chemical potential $\mu_{B}$ [99]. Another one and is the relation to the deconfinement of quarks where, instead of forming hadronic bound states, deconfinement gives rise to a so-called quark-gluon plasma [37]. For large values of $\mu_{B}$, it is not known if both transitions occur simultaneously or, alternatively, intermediate phases exist [100]. Many effective theories, however, fail to address these questions, since they do not include any confinement mechanism even if they correctly capture the essence of dynamical mass generation. As we show in the next section, our model presents such confinement-deconfinement phase transition between fractionally charged quasiparticles, allowing for the investigation of the interplay between the latter and chiral symmetry restoration in a simple setup.

\section{EMERGENT QUARKS AND MESONS}

In this section, we departure from the half-filled vacuum of the Jackiw-Rebbi model, and study the properties of the system when extra particles are added. In Sec. IV A, we dope the system in the ordered phase with one fermion, and find a regime where the latter fractionalized into two deconfined fermionic quasiparticles bound to topological defects of the rotor field. We calculate the static potential between them, and show how it changes from repulsive to attractive in a different parameter region. This attractive potential confine the quarklike emergent quasiparticles, forming mesons with integer charge. In Sec. IV B, we investigate these two regimes for a finite density of extra particles, establishing well-defined deconfined and confined phases in the thermodynamic limit. Finally, in Sec. IV C, we show how these are separated by a first-order phase transition, associated also to the restoration of chiral symmetry, and how these effects could be investigated by modifying a single experimental parameter.

\section{A. Confinement of fractionally charged quasiparticles}

As mentioned before, the fundamental fields of the QCD sector of the Standard Model correspond to fractionally charged fermion fields, the so-called quarks, coupled to bosonic Yang-Mills fields, the so-called gluons [1]. In contrast, the fundamental fields of our model, Eq. (6), are fermion fields with integer charges coupled to the constrained Néel and canting fields. As noted in the introduction, however, the renormalized quasiparticles of a strongly coupled QFT may sometimes differ completely from its fundamental constituents. As shown below, our QFT, Eq. (6), displays a Jackiw-Rebbi-like mechanism of fractionalization [29], whereby soliton configurations of the Néel field host localized fermions with a fractional charge $q= \pm e / 2$, which will play the role of quarks, allowing us to discuss various aspects of a confinement mechanism.

In Figs. 7(a) and 7(b), we present the MPS numerical results for the real-space configurations of the Néel lattice field $n_{z}(j)=(1 / 2 S)\left(\left\langle S_{2 j}^{z}\right\rangle-\left\langle S_{2 j-1}^{z}\right\rangle\right)$, and the integrated fermion charge above the half-filled vacuum $N(j)=$ $Q(j) / e=\sum_{i<j}\left(\left\langle c_{i}^{\dagger} c_{i}\right\rangle-\frac{1}{2}\right)$ when one extra fermion is introduced above half-filling. These figures show that the Néel field presents a kink-antikink pair that interpolates between the different SSB sectors, and that each of these topological solitons hosts a localized fermionic excitation with charge $q=e / 2$, henceforth referred to as a "quark" by the analogy with the fractionally charged fundamental fermion fields of QCD. We note that similar quasiparticles with charges $q=-e / 2$ would appear for dopings below half-filling, playing the role of "antiquarks," and that quark-antiquark pairs could appear in the vacuum due to thermal fluctuations, as they correspond to higher-energy states of the theory. The mechanism of charge fractionalization induced by topological defects is not restricted to high-energy physics, as it occurs as well in condensedmatter systems, such as the Su-Schrieffer-Heeger (SSH) electron-phonon model for polyacetylene [101]. In that case, the defects form in a otherwise dimerized phononic background, playing a similar role to the rotor fields considered here. In both cases, fractionalization takes place since, even if creating topological defects has an energy cost, this is still lower than rearranging the system to a new ordered pattern given by the new filling. Since each defect has an associated fermionic state with charge $\pm 1 / 2$ [29], it is more favorable for the extra particles to fractionalize and bound to the defects.

Contrary to the general situation in $(1+1)$ lattice gauge theories, which can only host confining phases [36], we can find regimes where quarks-antiquarks can be confined or deconfined depending on the microscopic parameters. In order to explore this phenomenon, let us remark that the distance of the pair of quarks of Fig. 7(b) is determined by the external pinning of the Néel solitons of Fig. 7(a). As described in more detail in Appendix F, we introduce an external potential that breaks explicitly the translational invariance and localizes the solitons, which would otherwise travel freely through the chain, at the desired positions. This pinning makes our fractionally charged quasiparticles static, such that we can discuss the analog of the static quark potential [102]. We note that it is a standard practice in lattice gauge theories to use this terminology whether or not the charges actually correspond to dynamical quarks [36]. Therefore, the static quark potential quantifies the interaction energy between any pair of static charges as their distance is modified, giving information about confinement not only in QCD, but in other effective models.

In our model, the static quark potential $V_{1}(d)=E_{1}(d)$ $-E_{0}$ can be obtained using the MPS numerics by 
(a)

$g S / t=0.9$

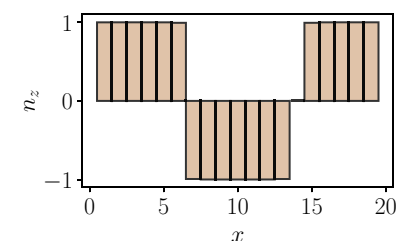

(b)

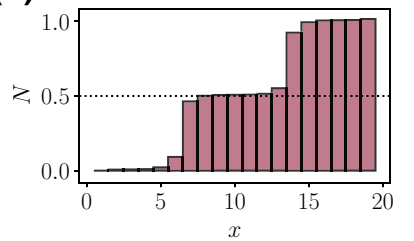

(c)

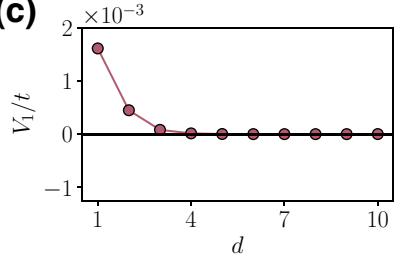

(d)

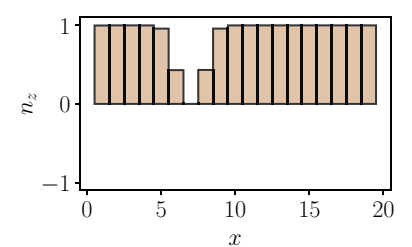

(e)

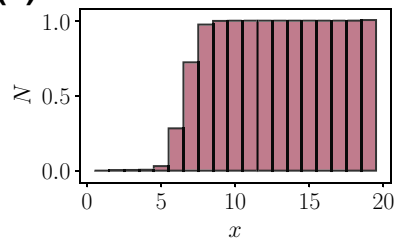

(f)

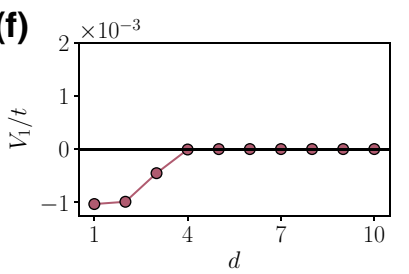

FIG. 7. Fractionalization and confinement: (a) space dependence of $n_{z}$ in the ground state of a chain with $N_{s}=41$ sites and $N_{f}=22$ fermions, for $h_{\ell} S / t=0.4$ and $h_{t} S / t=0.01$. Two solitons appear on the otherwise homogeneous configuration found at half-filling. (b) The accumulated particle density shows how each defect carries a fermion with fractional charge $e / 2$. (c) The solitons repel each other at short distances, implying that the composite soliton-charge quasiparticles are deconfined. (d) At larger values of $g S / t$, solitons merge forming a quark-bag quasiparticle with integer charge (e). (f) In this case, the potential energy between the solitons is attractive, indicating a transition to a confined phase.

calculating the energy $E_{1}(d)$ of the doped system with an extra fermion that fractionalizes into a pair of quarks pinned at a distance $d$, measured in unit cells $(2 a)$, with respect to the energy $E_{0}$ of a pair pinned far apart $(d \gg 1)$. We note that the standard situation of $(1+1)$ lattice gauge theories, such as the Schwinger model [24], is that the preservation of gauge symmetry requires that the static charges must be connected through an intermediate electric field string, such that the energy increases with the separation $d$ and leads to a linearly increasing static quark potential [103,104]. In our case, the situation is completely different, as the energy of the deformed Néel field that connects the two quarks in Fig. 7(c) is independent of the pinning distance, and confinement is thus not enforced $a$ priori. As argued below, there exists a competing mechanism that either favors confinement or deconfinement depending on the microscopic parameters of the model.

In Fig. 7(c), we depict the distance dependence of the static quark potential for the $S=1 / 2$ rotor Jackiw-Rebbi model for coupling $g S / t=0.9$, and setting the other parameters such that we lie in the chiral-SSB

phase. As can be observed in this figure, the potential decreases with the distance for small soliton separations, which means that the quarks repel each other. Hence, in the absence of the external pinning, the fractionally charged quasiparticles would move freely at large distances from each other, and thus appear as asymptotic excitations in the spectrum of the RJR QFT. As we increase the coupling to $g S / t=1.1$, Figs. 7(d) and 7(e) show that a completely different quasiparticle emerges. In this case, the Néel field no longer interpolates between the two SSB sectors, but is instead suppressed within a small region of space where, as shown in Fig. 7(e), an integer-charged fermion is localized. This situation is reminiscent of the so-called quark-bag models $[105,106]$, where quarks and gluons are locked within a finite region of space, in which a phenomenological term that compresses the bag compensates the outward pressure of the quarks that are held inside, and confinement results from the competition of these two terms. The present situation is closer in spirit to the soliton quark model $[107,108]$, where the fermions deplete a SSB condensate in a finite region of space, gaining kinetic energy at the expense of the cost of deforming the condensate. In our case, as the Néel field vanishes in the inner region, the scalar condensate will become zero $\Sigma_{0}(x)=0, \forall x \in$ $\left[x_{0}-\xi, x_{0}+\xi\right]$, such that the bound fermions have a vanishing dynamically generated mass, increasing their kinetic energy and the outward pressure. As mentioned above, this is compensated by the energy cost due to the inhomogeneous layout of the Néel field and the accompanying scalar condensate.

To substantiate this neat picture and connect it to the quark confinement, we should provide evidence that the integer-valued charges shown in Fig. 7(e) are the result of an attractive force between the fractionally charged quasiparticles of Figs. 7(a) and 7(b). This evidence of confinement is supported by the numerical results presented in Fig. 7(f), where we show that the static quark potential increases with the interquark distance in this case. Hence, as advanced in the introduction, it is possible to understand the microscopic confinement mechanism in our model. In the regime $g<g_{c}$, the outward pressure of the quarks overcomes the inner force that tends to re-establish the homogeneity of the condensate, such that the quarks get deconfined and can move synchronous with the kink or antikink. This changes for $g>g_{c}$, where the inward force to re-establish condensate homogeneity prevails, and the quarks get confined within the so-called quark bag. For $h_{\ell} S / t=0.4$ and $h_{t} S / t=0.01$, we estimate a critical value of this confinement-deconfinement phase transition to be $g_{c} S / t \approx 1.01$ (see Appendix F).

We note that these integer-charged excitations also occur in other QFTs with a nonclassical scalar condensate due to chiral SSB, such as the Gross-Neveu model [105]. However, to the best of our knowledge, there is no deconfinement transition where they become a pair 
of fractionally charged fermions and, additionally, they require at least two fermion flavors to exist, which anyway masks the occurrence of fractionalization as happens for polyacetilene [101,109]. In cold-atom realizations of our model, both the confined and the deconfined regimes can be detected via in situ measurements of the atomic density distribution, where pinning centers should be added first to localize the extra particles. If the average density of bosonic atoms above half-filling is low enough, the localized quasiparticles will be well separated. In that case, the density distribution of the bosonic atoms will reveal fractionalization even in the absence of a quantum gas microscope.

\section{B. Quark crystals and chiral symmetry restoration}

Let us now move towards finite densities, and explore the RJR ground-state properties in the confined and deconfined regimes. In the deconfined regime, we show that the isolated quarks repel each other, such that they will maximize the interquark distance and broaden the density profiles if left unpinned. Accordingly, for finite fermion densities above the half-filled vacuum, one would expect the formation of a crystalline structure of equidistant kinks and antikinks with the corresponding periodic distribution of fractionally charged fermions, namely a quark crystal.

On the other hand, in the confined regime, we have no a priori intuition of the possible ground-state ordering. To gain such intuition, let us first calculate the static potential between two distant quark-bag excitations, each of which contains a pair of confined quarks and thus an integercharged fermion. The static bag potential can be obtained using the MPS numerics by considering in this case the energy of the system doped with a pair of fermions that are held inside the bags $E_{2}(d)$, and pinned at a distance $d, V_{2}(d)=E_{2}(d)-E_{0}$, where $E_{0}$ is again the energy of two quark bags pinned far apart. In order to fix the quarkbag distance as depicted in Fig. 8(a), we impose again an external potential that localizes them at two given locations (see Appendix F). Our numerical results show that the static fermion-bag potential of Fig. 8(b) increases with the interbag distance, proving that the quark bags attract each other. Therefore, if left unpinned, we expect that the two bags will merge yielding a wider depletion region of the condensate that can accommodate two integer-charged fermions, each of which can be thought of being composed of two confined quarks. This trend can be generalized to finite-density regimes, where we expect the appearance of an extensive quark bag that is sufficiently wide to host all of the extra fermions. In the context of ultracold atoms, this phase can be understood as a phase-separation phenomenon.

Let us now confirm this intuition by presenting the MPS numerics for the finite-density regime. In Figs. 9 (a) and 9(c), we display the real-space dependence of
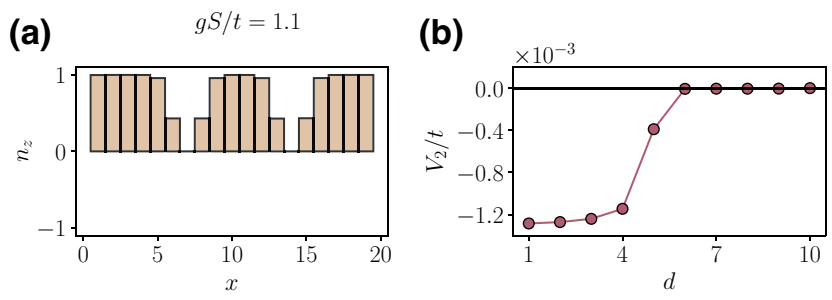

FIG. 8. Static quark-bag potential: (a) real-space configuration of the Néel field $n_{z}(x)$ in the ground state of a chain with $N_{s}=41$ sites and $N_{f}=23$ fermions, this is, two fermions above half-filling, for $h_{\ell} S / t=0.4$ and $h_{t} S / t=0.01$. The two quarkbag quasiparticles are pinned at a certain distance $d$ (see main text). (b) The potential energy between them decreases with the distance, and the minimum is reached when they are located at neighboring sites.

the scalar condensate for the deconfined and confined regimes without the pinning potentials. As can be clearly observed, Fig. 9(a) presents a periodic sequence of kinks and antikinks, each of which hosts a single localized quark, giving rise to the aforementioned quark crystal. On the other hand, as we increase the coupling strength, Fig. 9(c) displays an extensive region where the Néel field vanishes, and the dynamical fermionic mass is screened to zero. This wide bag accommodates for all the extra fermions, leading to a phase separation with respect to the region where the vacuum displays a large dynamically generated mass inhibiting the penetration of the massless confined quarks.

We note that the corresponding phases can be quantitatively distinguished by means of the static spin structure factor $S(k)=\left(1 / N_{s}^{2}\right) \sum_{i, j}\left\langle S_{i}^{z} S_{j}^{z}\right\rangle e^{i k(i-j)}$, which will peak at different momenta $k_{D}, k_{C}$ for the deconfined and confined phases. For the deconfined quark crystal, Fig. 9(b) shows that the corresponding peak of the structure factor occurs for a momentum that is commensurate with the fermionic density modulation of the scalar condensate $k_{D}=2 \pi N_{f} / N_{s}$. Conversely, for the confined phaseseparated bag phase, Fig. 9(d) shows that the peak at $k_{D}$ vanishes, and one gets instead a nonzero structure factor around $k_{C}=\pi$, signaling Néel order, which is partially broadened by the condensate deformation due to the quark bag. The inset of both figures displays the finite-size scaling of each peak, where we increase both the size $N_{s}$ and the number of fermions $N_{f}$, such that the density $\rho=N_{f} / N_{s}$ remains fixed. The extrapolated nonzero values of the corresponding peaks for $1 / L \rightarrow 0$ show that the quark-crystal and phase-separated bag phases are both stable in the thermodynamic limit. The characteristic properties of the system in both regimes can be detected in cold-atom realizations by measuring the in situ density distribution of the fermions using standard techniques. In our cold-atom QS this would correspond to measuring the density of bosonic atoms. The confined phase will be signaled by a larger density of the fermions at the position of 


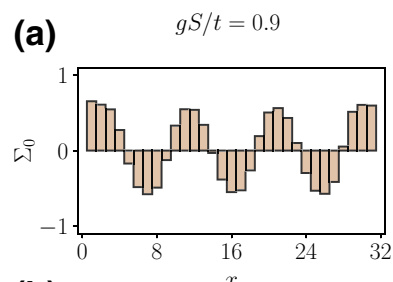

(b)
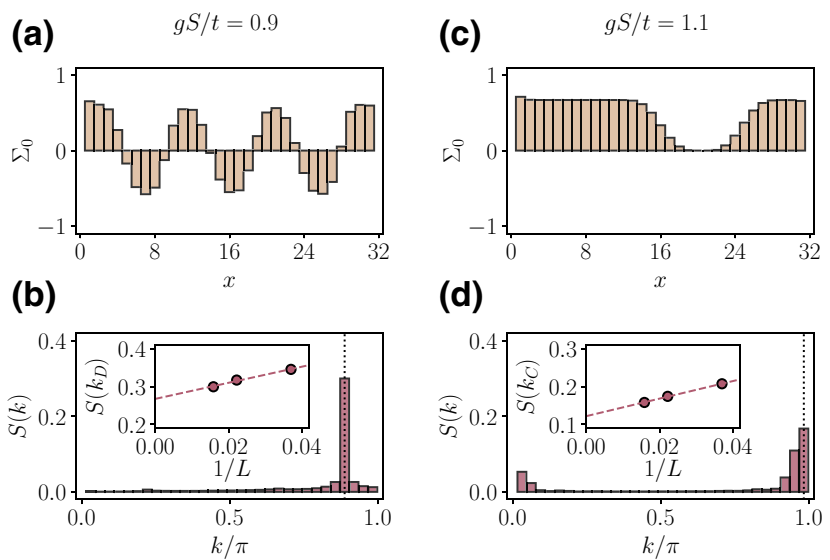

(d)

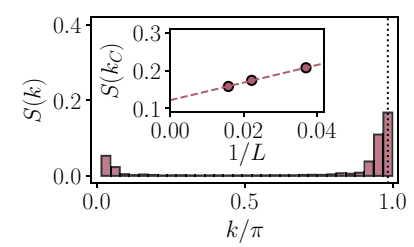

FIG. 9. Quark crystal and phase separation: (a) space dependence of the scalar condensate $\Sigma_{0}$ in the ground state of a chain with $N_{s}=63$ sites and $N_{f}=35$ fermions, for $h_{\ell} S / t=0.4$ and $h_{t} S / t=0.01$. For a finite density of particles above half-filling, the deconfined quarks rearrange forming an ordered structure. (b) Long-range order can be detected using the structure factor $S(k)$, which shows a peak at a momentum commensurate with the fermionic density, $k_{D} / \pi=2 N_{f} / N_{s}$. (c) In the confined phase, the attractive quark bags create an extensive region where the dynamical fermionic mass is screened to zero. (d) In this case, the peak at $k_{D}$ disappears, while $S(k)$ is nonzero around $k_{C}=\pi$, signaling a reduced but nonvanishing AF order. The insets show the finite-size scaling of the peak in $S(k)$ for each case, where the density is kept fixed.

the attractive quark bags, while in the deconfined phase a charge-density wave develops. In both cases a small symmetry-breaking term is required in order to pin the spatial density modulation. Moreover, a reliable detection of the wavevector of the charge-density wave in the deconfined phase requires a precise control over the average filling.

The vanishing value of the structure factor at momentum $k=\pi$ in the quark crystal suggests the possibility of a zero-temperature quantum chiral symmetry restoration for finite dopings - since this quantity is equivalent to the Néel order parameter $n_{z}$ used in our discussion of thermal chiral symmetry restoration for the RJR vacuum. This is confirmed by calculating the average value of the fermionic condensate $\bar{\Sigma}_{0}=\left(1 / N_{s}\right) \sum_{i} \Sigma_{0}(i)$, where we get $\bar{\Sigma}_{0}=0.06$ and $\bar{\Sigma}_{0}=0.48$ for the parameters used in Figs. 9(a) and 9(b), respectively. In this case, it is not the thermal fluctuations, but instead the finite density of topological solitons, which reduces the average value of the fermionic condensate to zero (up to finite-size effects), indicating that chiral symmetry is restored in the quarkcrystal phase. In this phase, therefore, chiral symmetry coexists with deconfinement. The situation is analogous in QCD, where both properties appear in the quark-gluon plasma. The presence of a single transition from this phase to a confined symmetry-broken phase, or the possibility of

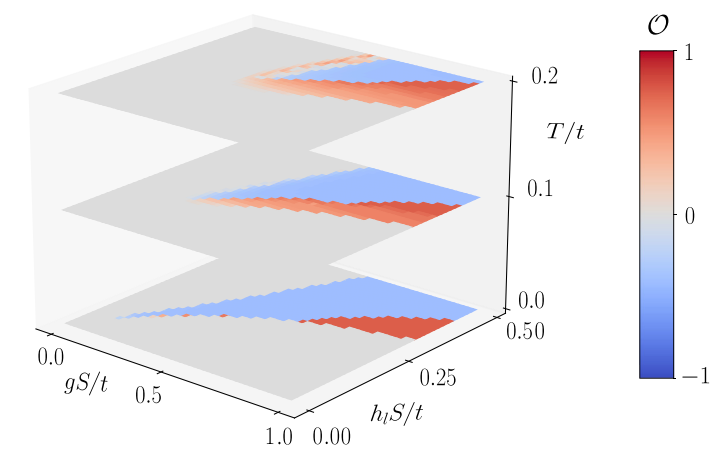

FIG. 10. Deconfinement and chiral symmetry restoration: phase diagram for different values of $T / t$ obtained in the HF approximation for a periodic chain with $N_{s}=64$ sites and $N_{f}=$ 35 fermions, with $h_{t} S=0.01 t$. The order parameter $\mathcal{O}$ (see main text) allows us to distinguish between the three different phases that appear for a finite doping. As the temperature increases, the phase transition line between the quark-bag and the quarkcrystal phases gets modified, until both of them disappear at a sufficiently high value of $T / t$.

intermediate phases with only one of these properties, is still an open question in particle physics [100]. The investigation of such interplay in simple models could help to gain a better understanding of it in more complicated theories, specially in regimes where the chemical potential is large and Monte Carlo simulations suffer from the sign problem [99].

Let us thus explore this interplay in the presence of thermal fluctuations. Figure 10 depicts the HF phase diagram for a finite density of fermions over the half-filled vacuum for different values of $T / t$. To distinguish the two phases, we use as an order parameter the difference between the structure factor at the two different momenta of the confined and deconfined phases, $\mathcal{O}=S\left(k_{C}\right)-S\left(k_{D}\right)$. This quantity is zero in the disordered paramagnetic phase, which corresponds to a Luttinger liquid, as in the vacuum case. Positive and negative finite values of $\mathcal{O}$ corresponds, on the other hand, to the quark-bag and quark-crystal phases, respectively. For $T / t=0$, we clearly distinguish these three phases. Note that, it is only in the ordered phases where the notion of confinement and deconfinement of fractionally charged quasiparticles is well defined. Within this region, we observe that both the quark-bag and the quark-crystal phases have finite extensions, with a phase transition line separating them. The ordered region shrinks as the temperature increases (Fig. 10). It is interesting to notice that, in our model, the quark crystal disappears more rapidly than the quark-bag phase.

\section{Confinement-deconfinement phase transition}

As we show in the previous sections, a characteristic feature of our $(1+1)$-dimensional QFT, Eq. (6), is the possibility to understand its confinement mechanism 

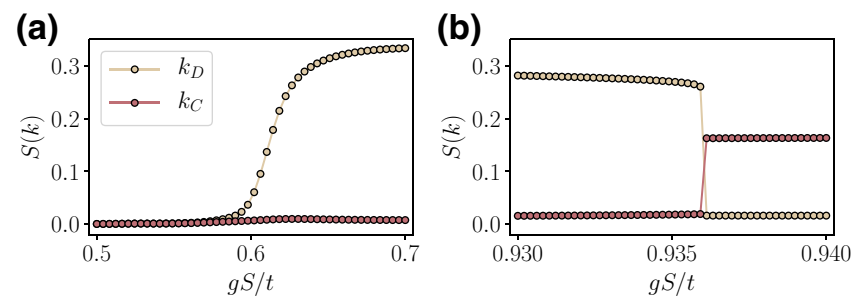

FIG. 11. Deconfinement phase transitions: structure factor at $k_{D}=2 \pi N_{f} / N_{s}$ and $k_{C}=\pi$ as a function of $g S / t$ for a chain with $N_{s}=63$ sites and $N_{f}=35$ particles, at $h_{\ell} S=0.4 t$ and $h_{t} S=0.01 t$. (a) In this case, $S\left(k_{D}\right)$ serves as an order parameter, signaling a direct transition between a disorder LL and an ordered quark crystal, while $S\left(k_{C}\right)$ remains zero all the way. (b) At higher values of $g S / t$, we observe a deconfinement-confinement transition between the two ordered phases, the quark crystal and the quark bag, where both $S\left(k_{D}\right)$ and $S\left(k_{C}\right)$ are the respective order parameters.

microscopically and, moreover, the existence of a deconfinement quantum phase transition as we vary the microscopic parameters. In order to study the latter in more detail, we make use again of the static structure factor peaks, which serve as order parameters to detect the corresponding phase transitions. In this section, we study two different types of phase transitions occurring at finite densities with DMRG, confirming the results obtained above using the HF method. The first one, corresponding to Fig. 11(a), describes the transition between the quark crystal and a longitudinal paramagnetic phase. As shown in this figure, following the spin-structure factor at the two characteristic momenta $k_{D}$ and $k_{C}$, one can see that we start from a disordered phase at small interactions, where both $S\left(k_{D}\right)$ and $S\left(k_{C}\right)$ are zero. As $g S / t$ increases, the quarkcrystal order parameter reaches a nonzero value $S\left(k_{D}\right)>0$, while $S\left(k_{C}\right)$ remains zero. This phase transition is continuous, similarly to the direct order-disorder transition we find for half-filling. For larger values of $g S / t$, we find another phase transition, again in correspondence with the HF results. Figure 11(b) shows the transition between the deconfined quark crystal $S\left(k_{D}\right)>0, S\left(k_{C}\right)=0$ towards the confined quark-bag phase $S\left(k_{D}\right)=0, S\left(k_{C}\right)>0$. In this case, this deconfinement transition is a first-order phase transition. This is believed to be the case also for the confinement-deconfinement transition in QCD at finite chemical potential. For $h_{\ell} S / t=0.4$ and $h_{t} S / t=0.01$, this transition is located at $g_{c} S / t=0.94$, which roughly agrees with the prediction we obtain using the static quark potential (i.e., $g_{c} S / t=1.01$ ). The difference shows the presence of many-body effects in the case of a quark crystal, where the interaction between two quarks is influenced by the presence of a finite density of them. This agreement supports our claim that the mechanism behind the transition between a quark crystal and a quark-bag phase is the confinement of quarklike fractional quasiparticles.

\section{CONCLUSIONS AND OUTLOOK}

In this work, we study several high-energy nonperturbative phenomena using a neat $(1+1)$ quantum field theory, the rotor Jackiw-Rebbi model, and propose a quantumsimulation scheme using a Fermi-Bose mixture of ultracold atoms in an optical lattice. Dirac fermions, whose interactions are mediated by spin- $S$ rotors in this model, acquire a dynamical mass through the spontaneous breaking of chiral symmetry. The generation of a mass term in the theory is accompanied here by antiferromagnetic order in the rotors, that we predict analytically in the large- $S$ limit of the continuum model. Using a lattice model that regularizes the theory, we study the phase diagram at half-filling in the presence of quantum and thermal fluctuation, showing how dynamical mass generation, in particular, survives in the ultimate quantum limit of $S=1 / 2$. We also show how, in this limit, the chiral symmetry-breaking quantum-phase transition lies in the Ising universality class and, moreover, we observe chiral symmetry restoration at sufficiently high temperatures.

We then focus on the regime of finite chemical potentials, where we find a confinement-deconfinement phase transition between quark bags and a crystal of fractional quarklike quasiparticles. This transition is characterized using the spin-structure factor, and its microscopic origin is uncovered by means of the static quark potential. We also show how deconfinement coexists with a restoration of chiral symmetry, even in the absence of thermal fluctuations. In this case, the latter occurs due to a proliferation of topological solitons at finite densities, drawing an interesting analogy to the quark-gluon plasma of QCD. Our results indicate how confinement between fractional charges could be further investigated in atomic experiments, shedding light onto one of the long-standing questions of particle physics.

In the future, it would be interesting to extend the large- $S$ techniques to a different family of lattice models that allow for symmetry-protected topological phases, and which have been studied so far for bosonic matter coupled to link spins in the $S=1 / 2$ limit [110-113], or for fermionic matter coupled to auxiliary scalar [114-116] or gauge $[53,117,118]$ fields, which can be substituted by the Néel and canting fields hereby introduced. These extensions would allow to explore the interplay of confinementdeconfinement transitions and topological phases of matter in a completely new framework. It would also be interesting to use matrix-product-state simulations to study real-time dynamics, serving as alternative benchmark for quantum simulations in addition to the finite-density regime hereby studied, where Monte Carlo simulations for a single flavor of fermion fields are expected to suffer from the sign problem. The model can also be easily extended to higher dimensions, where the simulation proposal can be generalized in a straightforward manner by using 
higher-dimensional optical lattices, which would also reach the limits of efficient tensor-network numerical techniques. It is precisely in the cases where numerical simulations show limitations where cold atoms represent an efficient alternative to provide a full solution of the quantum many-body problem.

\section{ACKNOWLEDGMENTS}

This project has received funding from the European Union Horizon 2020 research and innovation programme under the Marie Skłodowska-Curie Grant Agreement No. 665884, the Spanish Ministry MINECO and State Research Agency AEI (FIDEUA PID2019-106901GBI00/10.13039/501100011033, SEVERO OCHOA No. SEV-2015-0522 and CEX2019-000910-S, FPI), ERC AdG NOQIA, European Social Fund, Fundació Cellex, Fundació Mir-Puig, Generalitat de Catalunya (AGAUR Grant No. 2017 SGR 1341, CERCA program, QuantumCAT _U16-011424, co-funded by ERDF Operational Program of Catalonia 2014-2020), MINECO-EU QUANTERA MAQS (funded by The State Research Agency (AEI) PCI2019-111828-2/10.13039/501100011033), and the National Science Centre, Poland-Symfonia Grant No. 2016/20/W/ST4/00314. A.D. acknowledges the financial support from a fellowship granted by la Caixa Foundation (ID 100010434, fellowship code $\mathrm{LCF} / \mathrm{BQ} / \mathrm{PR} 20 / 11770012)$. A.B. acknowledges support from the Ramón y Cajal program RYC-2016-20066, and CAM/FEDER Project S2018/TCS-4342 (QUITEMAD$\mathrm{CM})$ and the Plan Nacional Generación de Conocimiento PGC2018-095862-B-C22. M.A. was supported by the Deutsche Forschungsgemeinschaft (DFG, German Research Foundation) via Research Unit FOR 2414 under Project No. 277974659 and Germany's Excellence Strategy EXC-2111 39081486 and by the European Union via the ERC Starting Grant LaGaTYb (Grant Agreement No. 803047).

\section{APPENDIX A: IMPLEMENTATION WITH COLD ATOMS}

We propose to realize the $S=\frac{1}{2}$ limit of the lattice Hamiltonian, Eq. (1), with a mixture of bosonic and spinfull fermionic atoms. Notice that the roles of bosonic and fermion degrees of freedom are interchanged here. In particular, we propose to use hardcore bosons to simulate the dynamical fermionic matter, while the fermionic ones are used to implement the rotor fields. As becomes clear below, this approach is motivated by the specific choice of bosonic and fermionic species, which presents a wellcharacterized Feshbach resonance that allows an accurate experimental control of the interspecies scattering, the crucial ingredient in our scheme.

\section{Bose-Fermi Hamiltonian}

We aim at realizing the following grand-canonical Hamiltonian for the Bose-Fermi mixture

$$
H=H_{b}+H_{f}+H_{b f},
$$

where $H_{b}$ describes the dynamics of the bosonic atoms of mass $m_{b}, H_{f}$ the one of the fermionic atoms of mass $m_{f}$, and $H_{b f}$ the interaction between the two species. The bosonic part of the Hamiltonian is defined as follows:

$$
\begin{aligned}
H_{b}= & -t \sum_{i}\left(b_{i}^{\dagger} b_{i+1}+\text { h.c. }\right)+\frac{U}{2} \sum_{i} b_{i}^{\dagger} b_{i}^{\dagger} b_{i} b_{i} \\
& -\sum_{i} \mu_{i} b_{i}^{\dagger} b_{i},
\end{aligned}
$$

where $b_{i}$ and $b_{i}^{\dagger}$ are the bosonic creation and annihilation operators acting on lattice site $i$. This grand-canonical Hamiltonian describes the tunneling of bosonic atoms in a 1D lattice with strength $t$, and the Hubbard interactions with energy $U$. Here, $\mu_{i}=\mu-V_{b, i}$ is expressed in terms of the chemical potential $\mu$ and the on-site optical trapping potential $V_{b, i}$, and controls the bosonic filling in the local density approximation. The fermionic contribution can be divided in two parts $H_{f}=H_{f}^{m}+H_{f}^{i}$, where the first term describes the external motional degrees of freedom

$$
\begin{aligned}
H_{f}^{m}= & -t_{f} \sum_{i, \sigma}\left(f_{i, \sigma}^{\dagger} f_{i+1, \sigma}+\text { H.c. }\right)+U_{\uparrow \downarrow} \sum_{i} f_{i, \downarrow}^{\dagger} f_{i, \uparrow}^{\dagger} f_{i, \uparrow} f_{i, \downarrow} \\
& -\sum_{i} \mu_{i, \uparrow} f_{i, \uparrow}^{\dagger} f_{i, \uparrow}-\sum_{i} \mu_{i \downarrow} f_{i, \downarrow}^{\dagger} f_{i, \downarrow} .
\end{aligned}
$$

Here, $f_{i \sigma}$ and $f_{i \sigma}^{\dagger}$ are the fermionic creation and annihilation operators acting on lattice site $i$ with internal state $\sigma=$ $\{\uparrow, \downarrow\}$. Fermions are trapped in a very deep optical lattice, such that $t_{f} \ll t$, and we can neglect their tunneling along the 1D lattice during the timescale of interest. They interact with Hubbard interaction $U_{\uparrow \downarrow}$, and their filling is controlled by the local chemical potentials $\mu_{i \sigma}=\mu_{\sigma}-V_{f, i}$, where $\mu_{\sigma}$ is the chemical potential for the fermionic atoms in each internal state, and $V_{f, i}$ is an optical trapping potential. In addition, the internal degrees of freedom are described by

$$
H_{f}^{i}=\sum_{i, \sigma} \epsilon_{\sigma} f_{i, \sigma}^{\dagger} f_{i, \sigma}+\sum_{i}\left(\Omega_{d} \cos \omega_{d} t f_{i, \uparrow}^{\dagger} f_{i, \downarrow}+\text { H.c. }\right),
$$

where we introduce the atomic energy levels $\epsilon_{\uparrow}, \epsilon_{\downarrow}$ for the two states of the fermionic species, and a local driving of frequency $\omega_{d}$ that induces local oscillations between these two states with a Rabi frequency $\Omega_{d}$, where $\hbar=1$ henceforth. As becomes clear below, this driving stems 
from radio-frequency radiation, which has a negligible momentum, and one can thus neglect recoil effects that would couple the internal and external degrees of freedom. Finally, the interaction between the two species is

$$
\hat{H}_{b f}=\sum_{i} b_{i}^{\dagger} b_{i}\left(U_{b \uparrow} f_{i, \uparrow}^{\dagger} f_{i, \uparrow}+U_{b \downarrow} f_{i, \downarrow}^{\dagger} f_{i, \downarrow}\right),
$$

which describes the on-site interaction between bosonic and fermionic atoms, and depends on the internal spin state of the fermions, i.e., in general $U_{b \uparrow} \neq U_{b \downarrow}$. As is clear for the particular Bose-Fermi mixture discussed below, there are also boson-fermion scattering processes where the internal states of the fermions is changed by populating other bosonic states such that the total angular momentum along the quantization axis is conserved. Nonetheless, the corresponding Zeeman energies of the initial and final states of these so-called spin-exchange processes are not resonant in this system and, therefore, they are strongly suppressed.

Let us now discuss the steps to arrive at the desired model, Eq. (1), and find the relation between the model microscopic couplings and the cold-atom experimental parameters. Using two internal states $|\uparrow\rangle$ and $|\downarrow\rangle$ with different magnetic moments, the energy splitting corresponds to the Zeeman energy and can be tuned via an external magnetic. The driving frequency is near-resonant and fulfils $\omega_{d}=\left(\epsilon_{\uparrow}-\epsilon_{\downarrow}\right)-\Delta_{d}$, where $\Delta_{d}$ is the so-called detuning. Let us note at this point that the optical trapping potential $V_{f, i}$ of Eq. (A3) is assumed to be state independent, which is generally the case, such that it does not modify the above resonance condition, and need not be included in the present discussion. Moving to an interaction picture with respect to

$$
H_{f, 0}^{i}=\sum_{i, \sigma} \epsilon_{\sigma} f_{i, \sigma}^{\dagger} f_{i, \sigma}
$$

the fermionic operators become $f_{i, \sigma} \rightarrow f_{i, \sigma}(t)=f_{i, \sigma} e^{-i \epsilon_{\sigma} t}$. Furthermore, assuming that $\left|\Omega_{d}\right|,\left|\Delta_{d}\right| \ll \omega_{d}$, we can perform a rotating-wave approximation such that

$$
H_{f}^{i}(t) \approx \sum_{i}\left(\frac{\Omega_{d}}{2} e^{i \Delta_{d} t} f_{i, \uparrow}^{\dagger} f_{i, \downarrow}+\text { H.c. }\right) .
$$

Finally, by moving to a frame that rotates with the drive frequency, this Hamiltonian can be written as the following time-independent term

$$
\hat{H}_{f}^{i}=\sum_{i}\left[\frac{\Delta_{d}}{2}\left(\hat{f}_{i, \uparrow}^{\dagger} \hat{f}_{i, \uparrow}-\hat{f}_{i, \downarrow}^{\dagger} \hat{f}_{i, \downarrow}\right)+\frac{\Omega_{d}}{2}\left(\hat{f}_{i, \uparrow}^{\dagger} \hat{f}_{i, \downarrow}+\hat{f}_{i, \downarrow}^{\dagger} \hat{f}_{i, \uparrow}\right)\right] .
$$

where we define the Bloch sphere in a way that $\Omega_{d} \in \mathbb{R}$, i.e., we use the phase of the driving as a reference for subsequent measurements. Here, we introduce the following relation between the fermionic operators in the original Schrödinger picture $f_{i, \sigma}$, and those $\hat{f}_{i, \sigma}$ in the rotating frame

$$
\begin{aligned}
& \hat{f}_{i, \uparrow}=f_{i, \uparrow} e^{-i\left(\epsilon_{\uparrow}-\Delta_{d} / 2\right) t}, \\
& \hat{f}_{i, \downarrow}=f_{i, \downarrow} e^{-i\left(\epsilon_{\downarrow}+\Delta_{d} / 2\right) t},
\end{aligned}
$$

We can now define the following spin- $\frac{1}{2}$ operators in terms of these fermionic annihilation and creation operators

$$
\begin{aligned}
& S_{i}^{z}=\frac{1}{2}\left(\hat{f}_{i, \uparrow}^{\dagger} \hat{f}_{i, \uparrow}-\hat{f}_{i \downarrow}^{\dagger} \hat{f}_{i, \downarrow}\right)=\frac{1}{2}\left(f_{i, \uparrow}^{\dagger} f_{i, \uparrow}-f_{i, \downarrow}^{\dagger} f_{i, \downarrow}\right), \\
& S_{i}^{x}=\frac{1}{2}\left(\hat{f}_{i, \uparrow}^{\dagger} \hat{f}_{i, \downarrow}+\hat{f}_{i, \downarrow}^{\dagger} \hat{f}_{i, \uparrow}\right)=\frac{1}{2}\left(f_{i, \uparrow}^{\dagger} f_{i, \downarrow} e^{i \omega_{d} t}+\text { H.c. }\right) .
\end{aligned}
$$

Therefore, to measure the spin operators discussed in the main text, one would have to lock the phase evolution to the one set by the source that drives the transition.

After these derivations, we should enforce that only 1 fermion resides at each lattice site, which can be adjusted by the filling, and maintained by working in the regime where $t_{f} \ll t$, thus suppressing double occupancies. In this case, we obtain the desired $S=\left\langle S_{i}^{z}\right\rangle=\frac{1}{2}$ limit. Moreover, since the fermion tunneling $t_{f}$ is negligible, superexchange processes stemming from virtual double occupancies occurring at order $t_{f}^{2} / U_{\uparrow \downarrow}$ are also negligible, and we can finally arrive at an effective description according to the following grand-canonical Hamiltonian

$$
\begin{aligned}
\hat{H}= & -t \sum_{i}\left(\hat{b}_{i}^{\dagger} \hat{b}_{i+1}+\text { h.c. }\right)+\frac{U}{2} \sum_{i} \hat{n}_{i}\left(\hat{n}_{i}-1\right) \\
& -\sum_{i} \mu_{i} \hat{b}_{i}^{\dagger} \hat{b}_{i},+g \sum_{i} \hat{n}_{i} S_{i}^{z}-\sum_{i}\left(h_{\ell} S_{i}^{z}+h_{t} S_{i}^{x}\right),
\end{aligned}
$$

where the boson operators are not altered by moving to the rotating frame $\hat{b}_{i}=b_{i}$. In the Hamiltonian above, the coupling constant is defined as

$$
g=2\left(U_{b \uparrow}-U_{b \downarrow}\right),
$$

and the external field given by the driving term

$$
h_{\ell}=-\Delta_{d}, \quad h_{t}=-\Omega_{d}
$$

the specifics of which are discussed below. The local chemical potential $\mu_{i}$ is adjusted to vary the filling of the bosonic atoms, which is homogeneous in the central region of the trap. The variation of the filling allows us to explore the different quasiparticle regimes discussed in the main text.

\section{Softcore bosons}

We can now address the final step in the derivation. Dispensing with the chemical potential, and working in 


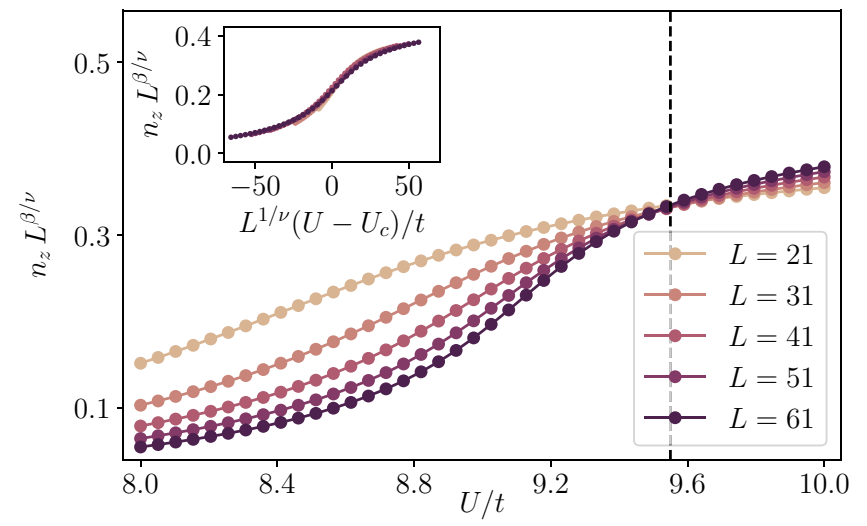

FIG. 12. Bosonic mass generation: order parameter $n_{z}$ in terms of $U / t$ for the ground state of a finite chain of different lengths $L$, with $g S / t=1.0, h_{\ell} S=0.5 t$, and $h_{t} S / t=0.05$. The different lines cross at the critical point, consistent with a phase transition in the Ising universality class $(v=1, \beta=1 / 8)$. The inset shows how the rescaled lines collapse into a single one.

the central region of the trap to neglect the residual onsite potentials, the Hamiltonian associated to Eq. (A11) coincides with the lattice model, Eq. (1), in the hardcore limit, $U / t \rightarrow \infty$, where bosons can be mapped to fermions via the Jordan-Wigner transformation. Nevertheless, we emphasize that the phases investigated in the main text appear also away from this singular point, i.e., for finite values of $U / t$, provided that the Hubbard interactions are sufficiently strong. This is true in particular for the AF phase found in the fermionic case, which survives away from the hardcore limit, giving rise to the generation of a dynamical mass for strongly correlated bosons. In Fig. 12, we represent the finite-size scaling for the Néel order parameter, and find that the disorder-order transitions occurs for interactions of the order $U_{c} \approx 10 t$. Below this value the softcore bosons are in a superfluid state, while the spins form a longitudinal paramagnet, similar to the disorder phase in the hardcore limit. Moreover, we find the the phase transition is also in the Ising universality class.

Once all the steps for the derivation of the target model, Eq. (A11), are discussed for a generic cold-atom setting, let us estimate the specific parameters for a mixture of bosonic ${ }^{87} \mathrm{Rb}$ and spinfull fermionic ${ }^{40} \mathrm{~K}$ atoms, and discuss the viability of the experimental realization. The following main ingredients are relevant for the implementation.

\section{Optical lattice potential}

The dominant transitions of the two alkali atoms, bosonic ${ }^{87} \mathrm{Rb}$ and fermionic ${ }^{40} \mathrm{~K}$ are

$$
\begin{aligned}
& \lambda_{\mathrm{Rb}, \mathrm{D} 2} \simeq 780 \mathrm{~nm}, \quad \lambda_{\mathrm{Rb}, \mathrm{D} 1} \simeq 795 \mathrm{~nm}, \\
& \lambda_{\mathrm{K}, \mathrm{D} 2} \simeq 767 \mathrm{~nm}, \quad \lambda_{\mathrm{K}, \mathrm{D} 1} \simeq 770 \mathrm{~nm},
\end{aligned}
$$

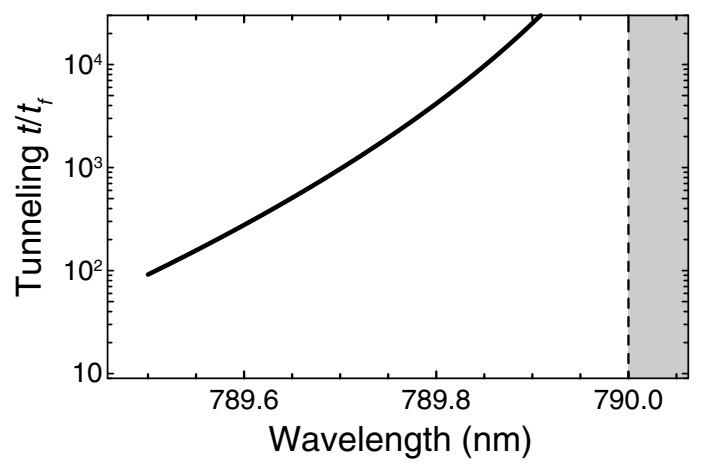

FIG. 13. Tunneling ratio $t / t_{f}$ as a function of the lattice wavelength. The zero crossing of the polarizability of $\mathrm{Rb}$ at $\lambda_{b z}$ is indicated by the dashed vertical line. For the numerical calculation, we choose a fixed laser intensity for the lattice, and tune it to a regime where $t_{f} / h \simeq 5\left\{\mathrm{mHz}\right.$. Since $t_{f}$ is more or less constant throughout the whole regime, $t / t_{f}$ illustrates the range of available tunneling couplings for $t$. This can be optimized further by going to shallower lattice depths. The gray region indicates a regime where the underlying optical potential is not suited to trap both species at the same position.

which allows for widely tunable polarizabilities and optical-lattice potentials. In order to achieve a large separation of timescales, it is desirable to achieve a large ratio of the tunneling couplings $t / t_{f}$, where $t$ is the strength of the tunnel coupling for the bosonic species, and $t_{f}$ denotes the spin-independent tunneling amplitude of the fermionic species. In essence, this means that the lattice potential experienced by the bosonic species should be much weaker compared to the one seen by the fermionic atoms. In order to reduce off-resonant photon scattering, which would result in additional heating, at the same time the detuning from any internal transition has to be maximized. Due to the large fine-structure splitting of $R b$, there is a convenient tuning range around the zero crossing of the polarizability at $\lambda_{b z}=790\{\mathrm{~nm}$. This range offers both a wide tunability of the tunneling ratio $t / t_{f}$ (Fig. 13) and a large detuning from all resonances to minimize heating.

\section{Tunable interspecies interactions}

In order to provide good control over the parameter $g$, Eq. (A12), that appears in the effective Hamiltonian, Eq. (A11), we propose to make use of the well-calibrated and easily accessible interspecies Feshbach resonance between the absolute ground states $\left|F=1, m_{F}=1\right\rangle$ of ${ }^{87} \mathrm{Rb}$ and $|\uparrow\rangle$ $\equiv\left|F=9 / 2, m_{F}=-9 / 2\right\rangle$ of ${ }^{40} \mathrm{~K}$ [119]. Although there does not seem to be a Feshbach resonance nearby to tune the scattering length between the ground state of Rubidium and $|\downarrow\rangle \equiv\left|F=9 / 2, m_{F}=-7 / 2\right\rangle$ of ${ }^{40} \mathrm{~K}$, the interaction parameter $g$ can still be fully tuned over a wide range of values as shown in Fig. 14, as it depends on the difference of scattering lengths. In this figure, we plot the scattering 


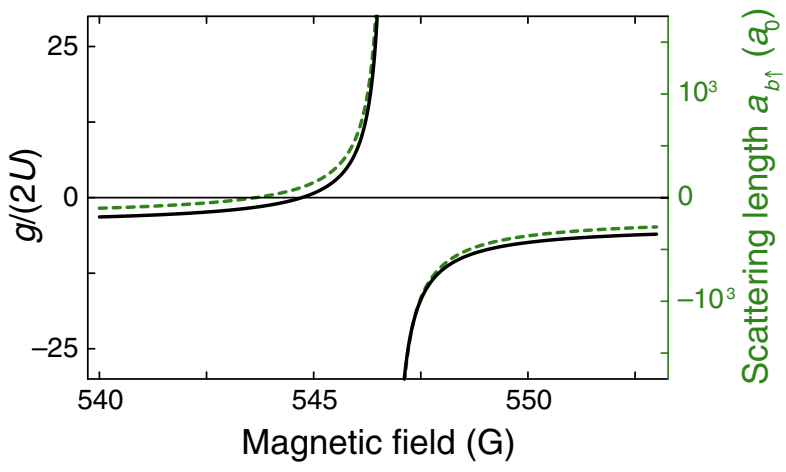

FIG. 14. Ratio of interactions strengths $g S / U$ as a function of magnetic field. The tunability here stems from the tunability of the interspecies scattering length $a_{b \uparrow}$, here shown in green and defined in Eq. (A15).

length $a_{b \uparrow}$ according to

$$
a_{b \uparrow}(B)=a_{b g}\left(1-\frac{\Delta}{B-B_{0}}\right)
$$

based on the theoretical values reported in Ref. [119], i.e., $B_{0}=546.75(6) \mathrm{G}, \Delta=-3.1 \mathrm{G}$, and $a_{b g}=-189 a_{0}$, where $a_{0}$ is the Bohr radius. The on-site interspecies interaction energy is determined by

$$
U_{b \sigma}=\frac{2 \pi \hbar^{2} a_{b \sigma}}{\mu_{b \sigma}} \int\left|w_{b}(\boldsymbol{r})\right|^{2}\left|w_{f}(\boldsymbol{r})\right|^{2} d^{3} \boldsymbol{r}
$$

where $\mu_{b \sigma}=m_{\mathrm{Rb}} m_{\mathrm{K}} /\left(m_{\mathrm{Rb}}+m_{\mathrm{K}}\right)$ is the reduced mass and $m_{\mathrm{Rb}}=86.9 u$ is the mass of one ${ }^{87} \mathrm{Rb}$ atom and $m_{\mathrm{K}}=$ $39.96 u$ the mass of one ${ }^{40} \mathrm{~K}$ atom; $u$ is the atomic mass unit. The functions $w_{b}(\boldsymbol{r})$ and $w_{f}(\boldsymbol{r})$ denote the Wannier functions of $\mathrm{Rb}$ and $\mathrm{K}$, respectively, which are different because the two species see a lattice potential of different depth. Through these expressions, we can obtain the parameter $g$, as discussed below.

\section{Interaction ratio $g S / U$}

Rubidium in its absolute ground state has a scattering length of $a_{b} \simeq 100.4 a_{0}$, and the on-site Hubbard interaction is defined as

$$
U=\frac{4 \pi \hbar^{2} a_{b}}{m_{\mathrm{Rb}}} \int\left|w_{b}(\boldsymbol{r})\right|^{4} d^{3} \boldsymbol{r}
$$

Neglecting the contribution from the Wannier functions, which is dependent on the lattice depth, we can achieve a wide tunability as illustrated in Fig. 14, where we plot $g S / U \approx a_{b \uparrow}\left(m_{\mathrm{Rb}}+m_{\mathrm{K}}\right) / 2 a_{b} m_{\mathrm{K}}$ as a function of the external magnetic field.

\section{Coupling between spin states}

Let us now discuss the specifics of the driving term discussed previously. The external fields $h_{\ell}, h_{t}$ can be realized with a radio-frequency or two-photon microwave transitions at frequency $\omega_{d}$ almost resonant with the Zeeman energy difference $\Delta E_{\uparrow, \downarrow}^{Z}=\epsilon_{i, \uparrow}-\epsilon_{i, \downarrow}$ between $|\uparrow\rangle$ and $|\downarrow\rangle$ atoms. For the Feshbach resonance shown in Fig. 14, the resonance occurs at $B_{0}$, which corresponds to $\Delta E_{\uparrow, \downarrow}^{Z} / h \approx 80 \mathrm{MHz}$, where $h$ denotes Planck's constant. The energy offset $h_{\ell}$ is then realized by detuning the coupling frequency from resonance, i.e., $h_{\ell}=\Delta E_{\uparrow, \downarrow}^{Z}-$ $\omega_{d}$. With single-photon transitions, Rabi frequencies $\Omega_{d} / h$ of several $10 \mathrm{kHz}$ can be easily achieved, which corresponds to the regime $\left|h_{t}\right|=\left|\Omega_{d}\right| \gg t$. Moreover, the pair of states $|\uparrow\rangle$ and $|\downarrow\rangle$ is well isolated from the other internal states, even in the presence of this coupling. The energetically closest transition is between the levels $|\downarrow\rangle$ and $\left|F=9 / 2, m_{F}=-5 / 2\right\rangle$, which is detuned by approximately $5 \mathrm{MHz} \gg \Omega_{d} / h$.

For some of the proposed phenomena, it is desirable to tune the coupling in order to enter the regime where $h_{t}$ is on the order of the tunnel coupling $t$. The level of control one can achieve is limited by the stability of the magnetic field that defines the Zeeman shift $\Delta E_{\uparrow, \downarrow}^{Z}$. We calculate the detuning from resonance $\delta h_{\ell}$ that occurs due to an imperfect control of the external magnetic field (Fig. 15). It is demonstrated that the fluctuations in the external field can be suppressed below $3 \times 10^{-6}$, as reported in Ref. [120]. The most sensitive regime occurs for $h_{\ell}=0$, where the lower bound for $h_{\text {trans }}$ would be on the order of a few $100 \mathrm{~Hz}$, which coincides with typical experimental values for $t$. Note, that the typical timescale for these fluctuations is large compared to the duration of the experiment, hence, the detuning $\delta h_{\ell}$ can be assumed constant but will fluctuate between individual experimental realizations.

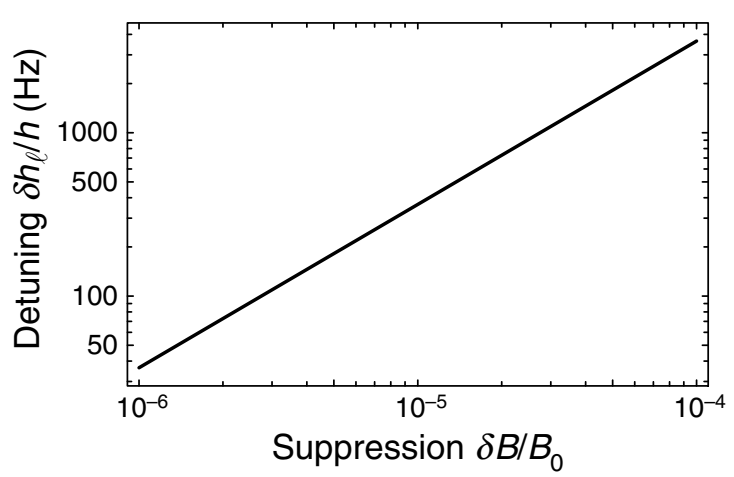

FIG. 15. Detuning of the microwave transition $\delta h_{\ell}$. The residual detuning is technically limited by magnetic field fluctuations $\delta B$. Estimating the residual detuning serves as a lower bound for the coupling strength $\omega_{d}$ and the control over the detuning $h_{\ell}$. 


\section{APPENDIX B: CONTINUUM LIMIT OF THE HAMILTONIAN LATTICE THEORY}

In this Appendix, we present the details for the derivation of the continuum Hamiltonian field theory for the rotor Jackiw-Rebbi model. Splitting the Hamiltonian in Eq. (1) as $H=H_{f}+H_{s f}+H_{s}$, the bare fermion tunneling $H_{f}$ leads to a periodic band structure with a pair of Fermi points at $\pm k_{F}= \pm \pi / 2 a$. Making the long-wavelength approximation

$$
c_{i} e^{-i k_{F} x_{i}} \sqrt{a} \psi_{+}(x)+e^{+i k_{F} x_{i}} \sqrt{a} \psi_{-}(x),
$$

yields a pair of slowly varying fields with the correct canonical algebra in the continuum limit $a \rightarrow 0$

$$
\left\{\psi_{\eta}(x), \psi_{\eta^{\prime}}\left(x^{\prime}\right)\right\}=\delta_{\eta, \eta^{\prime}} \frac{\delta_{x, x^{\prime}}}{a} \rightarrow \delta_{\eta, \eta^{\prime}} \delta\left(x-x^{\prime}\right) .
$$

By making a gradient expansion on the slowly varying fields $\psi_{\eta}(x+a)=\psi_{\eta}(x)+a \partial_{x} \psi_{\eta}(x)+\mathcal{O}\left(a^{2}\right)$, neglecting rapidly oscillating terms, and setting $a \sum_{j} \rightarrow \int d x$ in the continuum limit, the tunneling term becomes

$$
H_{f}=\int d x \sum_{\eta= \pm} \psi_{\eta}^{\dagger}(x)\left(-i \eta c \partial_{x}\right) \psi_{\eta}(x), \quad c=2 t a,
$$

where $c=2 t a$ plays the role of an effective speed of light. Defining the two-component spinors $\Psi(x)=$ $\left[\psi_{+}(x), \psi_{-}(x)\right]^{t}, \bar{\Psi}(x)=\left[\psi_{-}^{\dagger}(x), \psi_{+}^{\dagger}(x)\right]$, one readily sees that the Hamiltonian corresponds to a relativistic QFT of massless Dirac spinors

$$
H_{f}=\int d x \bar{\Psi}(x)\left(-i c \gamma^{1} \partial_{1}\right) \Psi(x)
$$

in a $(1+1)$ Minkowski spacetime $x^{0}=c t, x^{1}=x$ of metric $g_{\mu \nu}=\operatorname{diag}(1,-1)$, where $\gamma^{0}=\sigma^{x}, \gamma^{1}=-i \sigma^{y}$ are the gamma matrices $\left\{\gamma_{\mu}, \gamma_{\nu}\right\}=2 \delta_{\mu, \nu}$ in the so-called Weyl basis, and $\bar{\Psi}(x)=\Psi^{\dagger}(x) \gamma^{0}$. In the context of lattice gauge theories, the tunneling Hamiltonian corresponds to the staggered-fermion discretization of the Dirac equation [69] by setting $t=1 / 2 a$ and applying a Kawamoto-Smit rotation $c_{j} \rightarrow e^{i \pi j / 2} c_{j}$ [121].

Let us now turn our attention to the spin operators, which yield a $(2 S+1)$-dimensional representation of the $\mathfrak{s u}(2)$ algebra $\mathbf{S}_{i} \times \mathbf{S}_{j}=i \delta_{i, j} \mathbf{S}_{i}$. We introduce the so-called Néel $\mathbf{n}(x)$ and canting $\boldsymbol{\ell}(x)$ slowly varying fields

$$
\mathbf{S}_{i} \approx \cos \left(k_{N} x_{i}\right) S \mathbf{n}(x)+a \boldsymbol{\ell}(x),
$$

where the wave vector $k_{N}=\pi / a$ captures the Néel alternation of antiferromagnetic ordering. These operators satisfy the following algebra in the continuum limit:

$$
\begin{aligned}
& \ell(x) \times \mathbf{n}\left(x^{\prime}\right)=i \delta\left(x-x^{\prime}\right) \mathbf{n}(x), \\
& \ell(x) \times \boldsymbol{\ell}\left(x^{\prime}\right)=i \delta\left(x-x^{\prime}\right) \boldsymbol{\ell}(x), \\
& \mathbf{n}(x) \times \mathbf{n}\left(x^{\prime}\right)=i \delta\left(x-x^{\prime}\right) \ell(x)(a / S)^{2},
\end{aligned}
$$

where one must consider that there is a two-site unit cell, such that the continuum limit yields $\delta_{x, x^{\prime}} / 2 a \rightarrow \delta\left(x-x^{\prime}\right)$ in this case. In situations dominated by the Néel field, the contribution of the canting field will be negligible $\left\langle\boldsymbol{\ell}(a / S)^{2}\right\rangle \rightarrow \mathbf{0}$, and one obtains the algebra of position $\mathbf{n}$ and angular momentum $\boldsymbol{\ell}=\mathbf{n} \times\left(-i \nabla_{\mathbf{n}}\right)$ of a quantummechanical particle [58,59]. Moreover, in this limit, one also finds that

$$
\mathbf{n}(x) \cdot \mathbf{n}(x)=1+1 / S
$$

such that the particle will be confined to a unit sphere in the large-spin limit $S \gg 1$. Therefore, in the large- $S$ limit, the Néel and canting fields represent a the orientation of a quantum rotor and its angular momentum, respectively.

Combining the expressions for the Dirac, Eq. (B1), and rotor, Eq. (B5), fields, and neglecting again rapidly oscillating terms, we find that the spin-fermion coupling can be expressed as

$$
\begin{gathered}
H_{s f}=\int d x \bar{\Psi}(x) \mathbf{g}_{s} \cdot\left[\mathbf{n}(x)+\ell(x) \gamma^{0} / S\right] \Psi(x), \\
\mathbf{g}_{s}=g S \mathbf{e}_{z} .
\end{gathered}
$$

The first term can be understood as a Yukawa-like term that couples the fermion bilinear $\bar{\Psi}(x) \Psi(x)$ to the rotor projection $n_{z}(x)$ instead of the standard scalar field in a Yukawa theory [1]. The second term couples the timelike component of the fermion current $j^{0}(x)=\bar{\Psi}(x) \gamma^{0} \Psi(x)$, i.e., the charge density, to the projection of the rotor angular angular $\ell_{z}(x)$.

Finally, the continuum limit of the spin precession yields

$$
H_{s}=-\int d x \mathbf{h} \cdot \ell(x)
$$

In analogy to the original situation, Eq. (1), the rotor angular momentum is subjected to a magnetic field with both longitudinal and transverse components, being the latter responsible for introducing quantum fluctuations since $\left[\ell_{x}(x), n_{z}(x)\right] \neq 0$. Altogether, the continuum limit of the lattice model, Eq. (1) corresponds to the quantum field theory of Eq. (6).

\section{APPENDIX C: PATH-INTEGRAL FORMULATION OF THE CONTINUUM QFT}

In this Appendix, we provide a path-integral derivation of the continuum-limit rotor-fermion QFT, Eq. (6). This 
derivation serves for two purposes: on the one hand, it allows us to clarify the absence of a topological $\theta$ term for any spin $S$, differing markedly from $O(N)$ nonlinear sigma models arising from Heisenberg models [58]; on the other hand, it sets the stage for a large- $S$ limit approach to dynamical mass generation.

We are interested in the partition function $Z=$ $\operatorname{Tr}\left\{e^{-\beta H}\right\}$, where $H$ is the spin-fermion lattice Hamiltonian, Eq. (1). In the $\beta \rightarrow \infty$ limit of zero temperature, this partition function contains all the relevant information about quantum phase transitions related to the chiral SSB and dynamical mass generation we are seeking. Using fermionic and spin coherent states in Euclidean time $\tau=$ it $\in(0, \beta)$ [72], this partition function can be expressed as a functional integral in terms of anticommuting Grassmann fields for the fermions $\psi\left(\tau, x_{i}\right), \psi^{\star}\left(\tau, x_{i}\right)$ and commuting vector fields for the spins $\boldsymbol{\Omega}\left(\tau, x_{i}\right)$ lying in a two-sphere of radius $S$, which can be rescaled in terms of unit vector fields $\boldsymbol{\Omega}\left(\tau, x_{i}\right)=S \boldsymbol{\omega}_{i}(\tau)$. Using the resolution of the identity in terms of both types of fields [72], one finds that

$$
Z=\int D\left[\psi^{\star}, \psi, \omega\right] e^{-S_{E}\left[\psi^{\star}, \psi, S \omega\right]},
$$

where the Euclidean action $S_{E}=S_{s f}-i S A_{\mathrm{WZ}}$ can be expressed as a functional over the fermion and spin fields

$$
S_{E}=\int_{0}^{\beta} d \tau\left[\sum_{i} \psi^{\star}\left(\tau, x_{i}\right) \partial_{\tau} \psi\left(\tau, x_{i}\right)+H\left(\psi^{\star}, \psi, S \omega\right)\right],
$$

where $H\left(\psi^{\star}, \psi, S \omega\right)$ is obtained by substituting the fermion and spin operators in the normal-ordered Hamiltonian, Eq. (1) by the Grassmann and vector fields. One also finds the so-called Wess-Zumino term, which corresponds to the area enclosed by the trajectory of the spin field in the unit two-sphere

$$
A_{\mathrm{WZ}}=\int_{0}^{\beta} d \tau \int_{0}^{1} d s \sum_{i} \omega_{i}(s, \tau) \cdot\left[\partial_{s} \omega_{i}(s, \tau) \times \partial_{\tau} \omega_{i}(s, \tau)\right] .
$$

Here, this area is parametrized by $s \in[0,1]$, such that $\omega_{i}(s, 0)=\omega_{i}(s, \beta)$ correspond to the closed trajectories that cover a spherical cap $\Sigma$ between $\omega_{i}(0, \tau)=\omega_{i}(\tau)$ and the north pole $\omega_{i}(1, \tau)=\mathbf{e}_{z}$, which is used as the fiducial state in Bloch's sphere to define the spin coherent states (see Fig. 1).

In $O(3)$-symmetric situations, such as those arising in antiferromagnetic Heisenberg models, this Wess-Zumino term plays a crucial role as it is responsible for the mapping to a nonlinear sigma model with an additional topological $\theta$ term that depends on the integer or half-integer nature of the spins [58]. In the present case, however, there is no rotational symmetry in the classical Hamiltonian $H\left(\psi^{\star}, \psi, S \boldsymbol{\omega}\right)$, and one can see from the particular form of the spin-fermion coupling and the external field that it will suffice to use coherent states pointing along the meridian at vanishing longitude

$$
\boldsymbol{\omega}_{i}(\tau)=\omega_{i}^{x}(s, \tau) \mathbf{e}_{x}+\omega_{i}^{z}(s, \tau) \mathbf{e}_{z},
$$

which correspond to the great circle in the $x z$ plane of Fig. 1. Accordingly, $\omega_{i} \cdot\left(\partial_{s} \omega_{i} \times \partial_{\tau} \omega_{i}\right)=0$ and there is no enclosed area by the precession of the spins, such that the Wess-Zumino term vanishes $A_{\mathrm{WZ}}=0$.

We can now introduce the equivalent of the slowly varying quantum fields in Eqs. (B1) and (B5) in terms of the Grasmmann fermion fields

$$
\psi\left(\tau, x_{i}\right) e^{-i k_{F} x_{i}} \sqrt{a} \psi_{+}(\tau, x)+e^{+i k_{F} x_{i}} \sqrt{a} \psi_{-}(\tau, x),
$$

and the spin vector fields

$$
\omega_{i}(\tau) \approx \cos \left(k_{N} x_{i}\right) \mathbf{n}(\tau, x)+\frac{a}{S} \ell(\tau, x) .
$$

Proceeding in analogy to Appendix B, we perform the gradient expansion in the continuum limit $a \rightarrow 0$, neglect rapidly oscillating terms, and find $S_{E} \approx \int d^{2 x} \mathcal{L}(\mathbf{x})$ with the following Lagrangian density

$$
\begin{aligned}
\mathcal{L}(\mathbf{x})= & \bar{\Psi}(\mathbf{x})\left[\hat{\gamma}^{\mu} \partial_{\mu}+\mathbf{g}_{s} \cdot \mathbf{n}(\mathbf{x})\right] \Psi(\mathbf{x}) \\
& +\left[\mathbf{g} j_{0}(x)-\mathbf{h}\right] \cdot \ell(\mathbf{x}),
\end{aligned}
$$

with coupling $\mathbf{g}_{s}$ and external field $\mathbf{h}$ defined in Eqs. (B8) and (B9), respectively. Here, $\mathbf{x}=(c \tau, x)$ is a two-dimensional Euclidean space with metric $g_{\mu, v}^{E}=$ $\operatorname{diag}(1,1)$, and the Euclidean gamma matrices are $\hat{\gamma}^{0}=$ $\gamma^{0}, \hat{\gamma}^{1}=-i \gamma^{1}$. The Dirac spinor is composed of the rightand left-moving continuum Grassmann fields $\Psi(\mathbf{x})=$ $\left[\psi_{+}(\tau, x), \psi_{-}(\tau, x)\right]^{t}$, such that the adjoint becomes $\Psi(\mathbf{x})=\Psi^{\dagger}(\mathbf{x}) \hat{\gamma}^{0}=\left[\psi_{-}(\tau, x), \psi_{+}(\tau, x)\right]$. Likewise, the Néel and canting fields are the vector fields

$$
\begin{aligned}
& \mathbf{n}(\tau, x)=\frac{1}{2}\left[\omega_{2 i}(\tau)-\omega_{2 i-1}(\tau)\right], \\
& \ell(\tau, x)=\frac{S}{2 a}\left[\omega_{2 i}(\tau)+\omega_{2 i-1}(\tau)\right] .
\end{aligned}
$$

In the large- $S$ limit, and in situations dominated by Néel correlations $\left|\ell(a / S)^{2}\right| \rightarrow 0$, these fields are additionally 
subjected to the rotor constraints

$$
\mathbf{n}(\mathbf{x}) \cdot \mathbf{n}(\mathbf{x})=1, \quad \mathbf{n}(\mathbf{x}) \cdot \ell(\mathbf{x})=0,
$$

which can be included in the partition function

$$
Z=\int D[\bar{\Psi}, \Psi, \mathbf{n}, \ell] e^{-\int d^{2 x} \mathcal{L}(\mathbf{x})},
$$

through the functional integral measure $D[\bar{\Psi}, \Psi, \mathbf{n}, \ell]=$ $[(2 S+1) / 4 \pi] \prod_{\mathbf{x}} d \bar{\Psi}(\mathbf{x}) d \Psi(\mathbf{x}) d^{3} n(\mathbf{x}) d^{3} \ell(\mathbf{x}) \delta\left(\mathbf{n}^{2}-1\right)$ $\delta(\mathbf{n} \cdot \boldsymbol{\ell})$.

\section{APPENDIX D: EFFECTIVE ROTOR ACTION AND LARGE-S LIMIT}

In this Appendix, we give a detailed derivation of the effective rotor action, Eq. (9). As outlined in the main text, one can integrate out the Grassmann fields from the partition function

$$
Z=\int D[\mathbf{n}, \ell]\left(\int D[\bar{\Psi}, \Psi] e^{-\int d^{2 x} \mathcal{L}(\mathbf{x})}\right),
$$

since the corresponding functional integral reduces to a product of Gaussian integrals. These integrals are obtained after transforming the fields in terms of Matsubara frequencies $\omega_{n}=(\pi / \beta)(2 n+1)$, and quasimomentum $q a \in$ $[-\pi, \pi)$, according to

$$
\begin{aligned}
& \Psi\left(\tau, x_{i}\right)=\frac{1}{\sqrt{\beta L}} \sum_{n \in \mathbb{Z}} \sum_{q \in \mathrm{BZ}} e^{i\left(\omega_{n} \tau+q x_{i}\right)} \Psi\left(\omega_{n}, q\right), \\
& \bar{\Psi}\left(\tau, x_{i}\right)=\frac{1}{\sqrt{\beta L}} \sum_{n \in \mathbb{Z}} \sum_{q \in \mathrm{BZ}} e^{-i\left(\omega_{n} \tau+q x_{i}\right)} \bar{\Psi}\left(\omega_{n}, q\right) .
\end{aligned}
$$

The corresponding integrals lead to

$$
\begin{aligned}
\int & D[\bar{\Psi}, \Psi] e^{-\int d^{2 x} \mathcal{L}} \\
= & e^{-\int d^{2 x} \mathcal{L}_{r}} \prod_{n, q} \operatorname{det}\left[-i \omega_{n}+h_{D}\left(q, \mathbf{g}_{s} \cdot \mathbf{n}\right)\right],
\end{aligned}
$$

where we introduce a renormalized rotor Lagrangian

$$
\mathcal{L}_{r}=\left(\frac{\mathbf{g}}{2}-\mathbf{h}\right) \cdot \ell,
$$

for a homogeneous canting field $\ell(\mathbf{x})=\ell$. This term, Eq. (D4), which contains the original precession under the external field $-\mathbf{h}$, gets a contribution from the back action of the fermion current, Eq. (C7). For a homogeneous canting field, and at half-filling conditions $\int d x j_{0}(x)=N_{s} / 2$, this back action renormalizes the external field to $-(\mathbf{h}-$ $\mathrm{g} / 2$ ).
In Eq. (D3), we introduce the single-particle Hamiltonian of a $(1+1)$-dimensional Dirac fermion

$$
h_{D}(q, m)=c q \gamma^{5}+m \gamma^{0}
$$

with a Dirac mass proportional to the homogeneous Néel field $m=\mathbf{g}_{s} \cdot \mathbf{n}$. In the continuum and $T=0$ limits, the product of determinants involving these Dirac Hamiltonians can be expressed in terms of momentum integrals

$$
\prod_{n, q} \operatorname{det}\left[-i \omega_{n}+h_{D}\left(q, \mathbf{g}_{s} \cdot \mathbf{n}\right)\right]=e^{\left(1 / 4 \pi^{2} c\right) \int d^{2} k \log \left[k^{2}+\left(\mathbf{g}_{s} \cdot \mathbf{n}\right)^{2}\right]},
$$

where we introduce Euclidean momentum $\boldsymbol{k}=\left(\omega_{n}, c q\right)$.

This expression, together with Eq. (D3), leads to an effective action, which, up to an irrelevant term independent of the Néel and canting fields, reads as follows:

$$
\begin{aligned}
S_{\mathrm{eff}}= & \int d^{2} x\left\{\left(\frac{\mathbf{g}}{2}-\mathbf{h}\right) \cdot \boldsymbol{\ell}\right. \\
& \left.-\frac{\left(\mathbf{g}_{s} \cdot \mathbf{n}\right)^{2}}{4 \pi c}\left[\log \left(\frac{\Lambda_{c}}{\mathbf{g}_{s} \cdot \mathbf{n}}\right)^{2}+1\right]\right\},
\end{aligned}
$$

where the UV cutoff $\Lambda_{c}=2 t$ appears in the integrals over the Euclidean momentum $\int d^{2} k=\frac{2 \pi}{c} \int_{0}^{\Lambda_{c}} k d k$. Since the fields are homogeneous, this equation leads directly to the result, Eq. (9), used in the main text. This type of effective action, which contains a nonperturbative contribution $\sigma^{2} \log \sigma^{2}$, is a characteristic of the dynamical mass generation of the Gross-Neveu model [28], where $\sigma$ is an auxiliary field. In that case, the above calculation is equivalent to a large- $N$ limit summation of the leading Feynman diagrams, which contain a single fermion loop and all possible even numbers of legs for the $\sigma$ field [28]. This contributes to an effective potential that develops a doublewell structure as soon as $g \neq 0$, and thus induces the dynamical mass generation and chiral SSB. As noted in the main text, this SSB is dynamical as it requires quantummechanical effects via the fermion loops in order to take place, which makes it different from the classical SSB in the Jackiw-Rebbi model [29]. In the present case, Eq. (D7), the situation is different as the Néel and canting fields are not auxiliary, but have their own quantum dynamics that results from the noncommutativity of the rotor position and angular momentum. In particular, as a result of this competition, the dynamical SSB does not take place for an arbitrarily small $g$ regardless of the other microscopic parameters, but there will be critical lines that delimit the phase with a dynamically generated mass, as discussed in the main text.

Let us now describe the large- $S$ limit in more detail. Expressing the Néel and canting fields in the coherentstate basis, Eq. (10), one finds that the effective action, 
Eq. (D7), can be expressed in terms of an effective potential $S_{\text {eff }}=\beta L S V_{\text {eff }}\left(\theta_{A}, \theta_{B}\right)$, as there are no time derivatives of the fields. By letting $S \rightarrow \infty$, the solutions correspond to the saddle-point equations $\left.\partial_{\theta_{A}} V_{\text {eff }}\right|_{\theta^{\star}}=\left.\partial_{\theta_{B}} V_{\text {eff }}\right|_{\theta^{\star}}=$ 0 , which lead to the following system of nonlinear equations:

$$
\begin{aligned}
& \left\{\left(\frac{g}{2}-h_{\ell}\right)-\frac{t}{\pi S}\left(\frac{g S}{2 t}\right)^{2}\left(\sin \theta_{A}^{\star}-\sin \theta_{B}^{\star}\right) \log \left[\frac{4 t}{g S\left(\sin \theta_{A}^{\star}-\sin \theta_{B}^{\star}\right)}\right]^{2}\right\} \cos \theta_{A}^{\star}=-h_{t} \sin \theta_{A}^{\star}, \\
& \left\{\left(\frac{g}{2}-h_{\ell}\right)+\frac{t}{\pi S}\left(\frac{g S}{2 t}\right)^{2}\left(\sin \theta_{A}^{\star}-\sin \theta_{B}^{\star}\right) \log \left[\frac{4 t}{g S\left(\sin \theta_{A}^{\star}-\sin \theta_{B}^{\star}\right)}\right]^{2}\right\} \cos \theta_{B}^{\star}=-h_{t} \sin \theta_{B}^{\star} .
\end{aligned}
$$

Despite the nonlinearity, one can readily find an exact analytical solution to these saddle-point equations in the limit of vanishing transverse fields $h_{t}=0$, where the only possible solutions correspond to $\cos \theta_{A}^{\star}=\cos \theta_{B}^{\star}=0$. There are four possible solutions within $\boldsymbol{\theta}^{\star} \in[-\pi, \pi) \times$ $[-\pi, \pi)$, namely, as follows.

(i) North-pole paramagnet: this solution is $\theta_{A}^{\star}=\theta_{B}^{\star}=$ $\pi / 2$, where the canting field points towards the north pole $\ell(\mathbf{x})=(S / a) \mathbf{e}_{z}, \forall \mathbf{x}$. The corresponding spin coherent state is

$$
\left|g_{\ell P}^{+}\right\rangle=|S, S\rangle_{z, 1} \otimes|S, S\rangle_{z, 2} \otimes \cdots \otimes|S, S\rangle_{z, N_{S}}
$$

where $|S, m\rangle_{\alpha, i}$ is the common eigenstate of $\mathbf{S}_{i}^{2}, S_{i}^{\alpha}$ with eigenvalues $S(S+1)$ and $m \in\{-S,-S+1, \ldots, S-1, S\}$. Hence, all spin- $S$ particles are aligned towards the north pole. We note that this ordering is not caused by collective effects, but induced by the external longitudinal field, and that is why we refer to this state as a spin paramagnet.

(ii) South-pole paramagnet: this solution is $\theta_{A}^{\star}=\theta_{B}^{\star}=$ $-\pi / 2$, where the canting field points towards the south pole $\ell(\mathbf{x})=-(S / a) \mathbf{e}_{z}, \forall \mathbf{x}$. The corresponding coherent state is

$$
\left|g_{\ell P}^{-}\right\rangle=|S,-S\rangle_{z, 1} \otimes|S,-S\rangle_{z, 2} \otimes \cdots \otimes|S,-S\rangle_{z, N_{S}} .
$$

Hence, all spin- $S$ particles are aligned towards the south pole.

(iii) Néel antiferromagnets: these solutions are $\theta_{A}^{\star}=$ $-\theta_{B}^{\star}=\pi / 2$, or $\theta_{A}^{\star}=-\theta_{B}^{\star}=-\pi / 2$. In this case, and it is the Néel field that points towards the north $\mathbf{n}(\mathbf{x})=$ $\mathbf{e}_{z}, \forall \mathbf{x}$ and south pole $\mathbf{n}(\mathbf{x})=-\mathbf{e}_{z}, \forall \mathbf{x}$, respectively. The corresponding spin coherent states are

$$
\begin{aligned}
\left|g_{N}^{+}\right\rangle= & |S, S\rangle_{z, 1} \otimes|S,-S\rangle_{z, 2} \otimes \cdots \otimes \\
& \times|S, S\rangle_{z, N_{S}-1} \otimes|S,-S\rangle_{z, N_{S}}, \\
\left|g_{N}^{-}\right\rangle= & |S,-S\rangle_{z, 1} \otimes|S, S\rangle_{z, 2} \otimes \cdots \otimes \\
& \times|S,-S\rangle_{z, N_{S}-1} \otimes|S, S\rangle_{z, N_{S}} .
\end{aligned}
$$

Hence, all neighboring spin- $S$ particles are aligned in an antiparallel manner. We note that, in this case, this ordering is caused by collective effects, and the north- or south-pole solutions occur via the spontaneous symmetry breaking of the $\mathbb{Z}_{2}$ chiral symmetry $\mathbf{n}(\mathbf{x}) \rightarrow-\mathbf{n}(\mathbf{x})$ mentioned in the main text. This is why we refer to these states as antiferromagnets.

In order to decide which of the above orderings occurs in the system, we can compare the corresponding free energies per unit length $f\left(\theta_{A}^{\star}, \theta_{B}^{\star}\right)=(1 / L) F\left(\theta_{A}^{\star}, \theta_{B}^{\star}\right)=$ $-(1 / L \beta) \log Z\left(\theta_{A}^{\star}, \theta_{B}^{\star}\right)$, namely

$$
\begin{aligned}
& f\left( \pm \frac{\pi}{2}, \pm \frac{\pi}{2}\right)= \pm\left(\frac{g}{2}-h_{\ell}\right) \frac{S}{a}, \\
& f\left( \pm \frac{\pi}{2}, \mp \frac{\pi}{2}\right)=-\frac{t}{2 \pi a}\left(\frac{g S}{2 t}\right)^{2}\left[\log \left(\frac{2 t}{g S}\right)^{2}+1\right] .
\end{aligned}
$$

Comparing these energies, we find two critical lines $h_{\ell}^{ \pm}$that separate the Néel antiferromagnet from the two longitudinal paramagnets. The first one

$$
h_{\ell}^{-}=\frac{g}{2}-\frac{t}{\pi S}\left(\frac{g S}{2 t}\right)^{2}\left[\log \left(\frac{2 t}{g S}\right)+\frac{1}{2}\right],
$$

is obtained from $f(-\pi / 2,-\pi / 2)=f(+\pi / 2,-\pi / 2)$. Accordingly, for $h_{\ell}<h_{\ell}^{-}$, the ground state corresponds to the south-pole longitudinal paramagnet. Conversely, for $h_{\ell}>h_{\ell}^{-}$, we enter into the Néel antiferromagnet. The second line is

$$
h_{\ell}^{+}=\frac{g}{2}+\frac{t}{\pi S}\left(\frac{g S}{2 t}\right)^{2}\left[\log \left(\frac{2 t}{g S}\right)+\frac{1}{2}\right],
$$

and is obtained from $f(+\pi / 2,-\pi / 2)=f(+\pi / 2,+\pi / 2)$. In this case, for $h_{\ell}<h_{\ell}^{+}$, the ground state corresponds to the Néel antiferromagnet, while or $h_{\ell}>h_{\ell}^{+}$, we enter into the north-pole longitudinal paramagnet. Both critical 
(a)

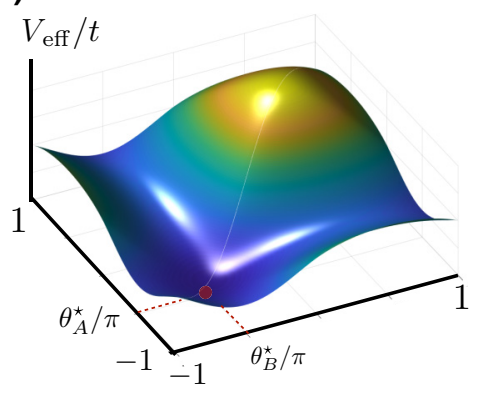

(b)

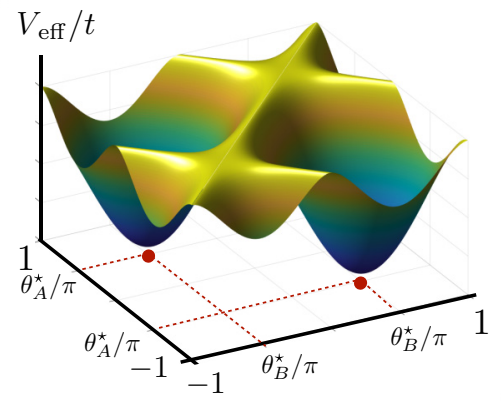

(c)

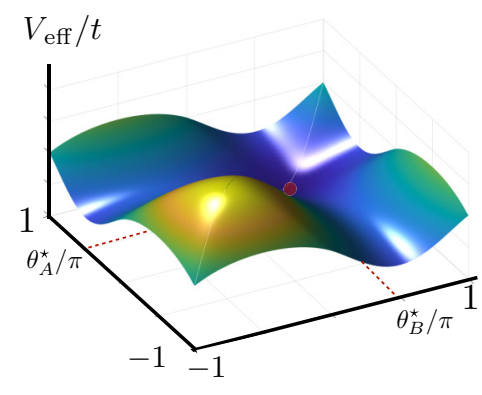

FIG. 16. Saddle points of the effective potential. (a) Effective potential for the south-pole paramagnetic regime $h_{\ell}=h_{\ell}^{-}-\delta / 2$, where $\delta=h_{\ell}^{+}-h_{\ell}^{-}$. (b) Effective potential for the Néel antiferromagnetic regime $h_{\ell}=h_{\ell}^{-}+\delta / 2$. (c) Effective potential for the north-pole paramagnetic regime $h_{\ell}=h_{\ell}^{-}+3 \delta / 2$.

lines correspond to a situation where the effective potential changes from a single minimum into a double well, as represented in Fig. 16.

(iv) Equator paramagnet: finally, before concluding this Appendix, let us discuss the other limit where one can find a different saddle-point solution. This corresponds to the $h_{\ell}=g=0$ and $h_{t}>0$ limit, where both Eqs. (D8) are solved when $\sin \theta_{A}^{\star}=\sin \theta_{B}^{\star}=0$. In this case, by comparing the corresponding free energies, one readily sees that there is a unique ground state corresponding to $\theta_{A}^{\star}=$ $\theta_{B}^{\star}=\pi$, where the canting field points along the equator $\ell(\mathbf{x})=(S / a) \mathbf{e}_{x}, \forall \mathbf{x}$. In terms of the spins, this phase corresponds to

$$
\left|g_{t P}\right\rangle=|S, S\rangle_{x, 1} \otimes|S, S\rangle_{x, 2} \otimes \cdots \otimes|S, S\rangle_{x, N_{s}}
$$

where all spins align parallel to the external transverse field. Since the ordering is not due to collective effects, we refer to this phase as a transverse paramagnet.

\section{APPENDIX E: SELF-CONSISTENT HARTREE-FOCK MEAN-FIELD THEORY}

In this Appendix, we discuss more in depth the details of the derivation of the self-consistent mean-field theory used in the main text. We start from the Hamiltonian of Eq. (1), and perform a Hartree-Fock decoupling of the spinfermion coupling

$$
\mathbf{g} \cdot \mathbf{S}_{i} n_{i} \simeq \mathbf{g} \cdot\left\langle\mathbf{S}_{i}\right\rangle n_{i}+\mathbf{g} \cdot \mathbf{S}_{i}\left\langle n_{i}\right\rangle-\mathbf{g} \cdot\left\langle\mathbf{S}_{i}\right\rangle\left\langle n_{i}\right\rangle
$$

where we introduce the number operator $n_{i}=c_{i}^{\dagger} c_{i}$. After the Hartree-Fock decoupling, the Hamiltonian can be expressed as the sum of noninteracting Hamiltonians for the fermion and spin sectors

$$
f=-\sum_{i} t c_{i}^{\dagger} c_{i+1}+\sum_{i} \epsilon_{f, i} n_{i}, \quad \epsilon_{f, i}=\mathbf{g} \cdot\left\langle\mathbf{S}_{i}\right\rangle
$$

$$
H_{s}=-\sum_{i} \mathbf{h}_{s, i} \cdot \mathbf{S}_{i}, \quad \mathbf{h}_{s, i}=\mathbf{h}-\mathbf{g}\left\langle n_{i}\right\rangle,
$$

where $f(s)$ stands for fermions (spins). Accordingly, the fermion tunnel in a potential-energy landscape $\epsilon_{f, i}$ set by the expectation value of the spins (see Fig. 1), while the spins precess in an effective external field, which becomes inhomogeneous depending on the average distribution of fermions.

This mean-field approximation requires the obsrevables $\left\{\left\langle\mathbf{S}_{i}\right\rangle,\left\langle n_{i}\right\rangle\right\}_{i=1}^{N_{s}}$ to be determined self-consistently, and we must deal with a number of self-consistency equations that grows linearly with the number of sites. The self-consistent loop consists in the following steps: we start by setting an initial spin configuration $\left\langle\mathbf{S}_{i}\right\rangle$, and compute the expectations values $\left\langle n_{i}\right\rangle$ by solving the fermionic tight-biding model, Eq. (E2), for a given temperature $T=\beta^{-1}$. These mean-field parameters are then used as input to determine the effective external field in the spin Hamiltonian, Eq. (E3), which is subsequently diagonalized, such that we can calculate the corresponding spin expectation values $\left\langle\mathbf{S}_{i}\right\rangle$ for a given temperature $T$.

This process must be iterated until reaching convergence for the free energy, which can be expressed as $F_{\mathrm{MF}}=F_{f}+$ $F_{s}+C$, where $F_{f}\left(F_{s}\right)$ is the free energy of the fermions (spins) and $C$ is the constant term appearing in the HartreeFock decoupling, Eq. (E1). The free energy for the system of fermions can be expressed in terms of the grand partition function

$$
Z_{f}=\operatorname{Tr}\left\{e^{-\beta\left(H_{f}-\mu N_{f}\right)}\right\},
$$

where we introduce the chemical potential $\mu$, and the total fermion number $N_{f}=\sum_{i} n_{i}$. For the mean-field decoupled model, this partition function can be readily expressed as

$$
Z_{f}=\prod_{n=1}^{N_{s}} Z_{f, n}, \quad Z_{f, n}=1+e^{-\beta\left(E_{f, n}-\mu\right)}
$$


where $E_{f, n}$ are the eigenvalues of the quadratic fermionic Hamiltonian, Eq. (E2). The free energy, which can be expressed as $F_{f}=-(1 / \beta) \log Z_{f}+(\mu / \beta)(\partial / \partial \mu) \log Z_{f}$, thus becomes

$$
F_{f}=-\frac{1}{\beta} \sum_{n} \log Z_{f, n}+\mu \sum_{n} n_{\mathrm{FD}}(\mu, \beta)
$$

where $n_{\mathrm{FD}}(\mu, \beta)=\mu \partial_{\mu} Z_{f, n} / \beta Z_{f, n}=1 /\left[e^{\beta\left(E_{f, n}-\mu\right)}+1\right]$ is the so-called Fermi-Dirac distribution. For the spins, the number of which is conserved, the free energy can be written in terms of the canonical partition function

$$
Z_{s}=\operatorname{Tr}\left\{e^{-\beta H_{s}}\right\} .
$$

For the mean-field decoupled system, the Hamiltonian, Eq. (E3), can be diagonalized for each spin independently

$$
Z_{s}=\prod_{i=1}^{N_{s}} Z_{s, i}, \quad Z_{s, i}=\sum_{n=1}^{2 S+1} e^{-\beta E_{s, n}}
$$

where $E_{s, n}$ are the the $(2 S+1)$ energies of the spins, which are identical for all sites of the chain. The corresponding free energy is

$$
F_{s}=-\frac{1}{\beta} \sum_{i} \log Z_{s, i}
$$

\section{APPENDIX F: STATIC QUASIPARTICLE POTENTIALS}

In this Appendix, we give further details on the calculation of the static potentials between fractional quarklike quasiparticles and between quark bags. In both cases, this is achieved by first pinning two of these quasiparticles to certain locations in the chain, separated by a distance $d$ measured in unit cells $(2 a)$. In the first case, since each of them is associated to a soliton in the Néel-ordered rotor field, we introduce a small parallel field

$$
H_{\epsilon}=-\sum_{i} \epsilon_{i}(d) S_{i}^{z}
$$

with

$$
\epsilon_{i}(d)= \begin{cases}\epsilon(-1)^{i} & 1 \leq i<i_{0} \\ \epsilon(-1)^{i+1} & i_{0} \leq i<i_{0}+d \\ \epsilon(-1)^{i} & i_{0}+d \leq i \leq N_{s} .\end{cases}
$$

This field breaks translational invariance and pines two solitons, and the associated fractional fermionic charges, to positions $i_{0}$ and $i_{0}+d$. In Fig. 17(a), we choose $i_{0}=$ $\left(N_{s}+1\right) / 3$ and $\epsilon=0.02 t$, and represent the static quark potential $V_{1}(d)=E_{1}(d)-E_{0}$, where $E_{1}(d)$ and $E_{0}$ are the
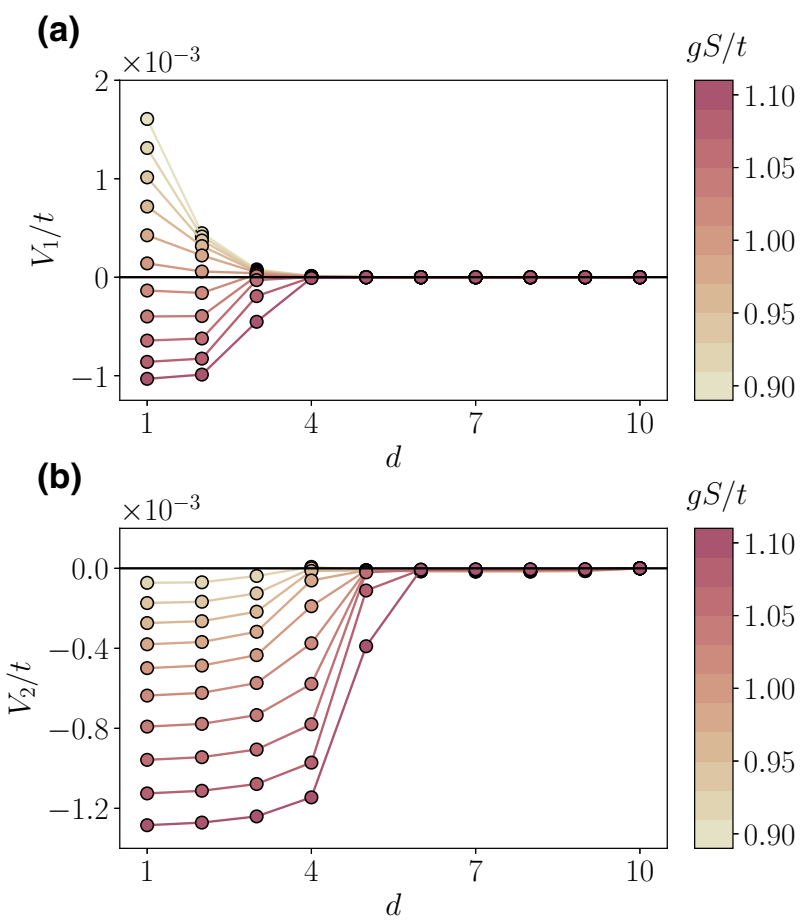

FIG. 17. Deconfinement transition and quark-bag instability: (a) potential energy between two defects pinned at a distance $d$ (see main text) for a chain with $N_{s}=41$ sites and $N_{f}=22$, at $h_{\ell} S=0.4 t, h_{t} S=0.01 t$ and $\epsilon S=0.02 t$. As $g S / t$ is increased, the repulsive potential between two solitons turns into an attractive one, signaling a deconfinement-confinement transition. (b) Attractive potential energy between two pinned soliton bags, for $N_{s}=41$ and $N_{f}=23$.

energies of a chain with $N_{s}$ sites and $N_{f}=\left(N_{s}+1\right) / 2+1$ fermions under the pinning potential, Eq. (F2), for a given $d$ and $i_{0}=10$, respectively. The potential changes from repulsive to attractive as we increase $g S / t$. For the parameters $h_{\ell} S=0.4 t, h_{t} S=0.01 t$, the transition is located at $g_{c} S / t \approx 1.01$.

The static potential between two quark-bag quasiparticles is obtained by first pinning them at a distance $d$, in a chain with $N_{s}$ sites and $N_{f}=\left(N_{s}+1\right) / 2+2$ fermions, using now a parallel field, Eq. (F1), with

$$
\epsilon_{i}=\epsilon\left[(-1)^{i}-2 \delta_{i, i_{0}}-2 \delta_{i, i_{0}+d}\right]
$$

The potential $V_{2}(d)=E_{2}(d)-E_{0}$, where $E_{2}(d)$ is now the corresponding energy when two quark bags are pinned at a distance $d$, is shown in Fig. 17(b) for different values of $g S / t$. In this case, the potential is always attractive.

[1] Michael E. Peskin and Daniel V. Schroeder, An Introduction to Quantum Field Theory (Addison-Wesley, Reading, USA, 1995). 
[2] X.-G. Wen, Quantum Field Theory of Many-Body Systems: From the Origin of Sound to an Origin of Light and Electrons (Oxford University Press, Oxford, 2007).

[3] L. Landau, On the theory of phase transitions, Zh. Eksp. Teor. Fiz 11, 26 (1937).

[4] L. Landau, The theory of a fermi liquid, Sov. Phys. JETP 30, 1058 (1956).

[5] P. W. Anderson, More is different, Science 177, 393 (1972).

[6] Kenneth G. Wilson and J. Kogut, The renormalization group and the expansion, Phys. Rep. 12, 75 (1974).

[7] Kenneth G. Wilson, The renormalization group: Critical phenomena and the Kondo problem, Rev. Mod. Phys. 47, 773 (1975).

[8] Timothy J. Hollowood, Renormalization Group and Fixed Points in Quantum Field Theory (Springer, Heidelberg, Germany, 2013).

[9] Kenneth G. Wilson, Confinement of quarks, Phys. Rev. D 10, 2445 (1974).

[10] John B. Kogut, An introduction to lattice gauge theory and spin systems, Rev. Mod. Phys. 51, 659 (1979).

[11] Leticia Tarruell, Daniel Greif, Thomas Uehlinger, Gregor Jotzu, and Tilman Esslinger, Creating, moving and merging Dirac points with a Fermi gas in a tunable honeycomb lattice, Nature 483, 302 (2012).

[12] L. Duca, T. Li, M. Reitter, I. Bloch, M. Schleier-Smith, and U. Schneider, An Aharonov-Bohm interferometer for determining Bloch band topology, Science 347, 288 (2015).

[13] N. Fläschner, B. S. Rem, M. Tarnowski, D. Vogel, D.-S. Lühmann, K. Sengstock, and Christof Weitenberg, Experimental reconstruction of the Berry curvature in a Floquet Bloch band, Science 352, 1091 (2016).

[14] Christian Schweizer, Fabian Grusdt, Moritz Berngruber, Luca Barbiero, Eugene Demler, Nathan Goldman, Immanuel Bloch, and Monika Aidelsburger, Floquet approach to $\mathbb{Z} 2$ lattice gauge theories with ultracold atoms in optical lattices, Nat. Phys. 15, 1168 (2019).

[15] Alexander Mil, Torsten V. Zache, Apoorva Hegde, Andy Xia, Rohit P. Bhatt, Markus K. Oberthaler, Philipp Hauke, Jürgen Berges, and Fred Jendrzejewski, A scalable realization of local $\mathrm{u}(1)$ gauge invariance in cold atomic mixtures, Science 367, 1128 (2020).

[16] Bing Yang, Hui Sun, Robert Ott, Han-Yi Wang, Torsten V. Zache, Jad C. Halimeh, Zhen-Sheng Yuan, Philipp Hauke, and Jian-Wei Pan, Observation of gauge invariance in a 71-site quantum simulator, arXiv:2003.08945 (2020).

[17] R. Gerritsma, G. Kirchmair, F. Zähringer, E. Solano, R. Blatt, and C. F. Roos, Quantum simulation of the Dirac equation, Nature 463, 68 (2010).

[18] R. Gerritsma, B. P. Lanyon, G. Kirchmair, F. Zähringer, C. Hempel, J. Casanova, J. J. García-Ripoll, E. Solano, R. Blatt, and C. F. Roos, Quantum Simulation of the Klein Paradox with Trapped Ions, Phys. Rev. Lett. 106, 060503 (2011).

[19] Esteban A. Martinez, Christine A. Muschik, Philipp Schindler, Daniel Nigg, Alexander Erhard, Markus Heyl, Philipp Hauke, Marcello Dalmonte, Thomas Monz, Peter
Zoller, and Rainer Blatt, Real-time dynamics of lattice gauge theories with a few-qubit quantum computer, Nature 534, 516 (2016).

[20] R. P. Feynman, Simulating physics with computers, Int. J. Theor. Phys. 21, 467 (1982).

[21] Maciej Lewenstein, Anna Sanpera, Veronica Ahufinger, Bogdan Damski, Aditi Sen(De), and Ujjwal Sen, Ultracold atomic gases in optical lattices: Mimicking condensed matter physics and beyond, Adv. Phys. 56, 243 (2007).

[22] Immanuel Bloch, Jean Dalibard, and Sylvain Nascimbène, Quantum simulations with ultracold quantum gases, Nat. Phys. 8, 267 (2012).

[23] R. Blatt and C. F. Roos, Quantum simulations with trapped ions, Nat. Phys. 8, 277 (2012).

[24] Julian Schwinger, Gauge invariance and mass. II, Phys. Rev. 128, 2425 (1962).

[25] N. S. Manton, The Schwinger model and its axial anomaly, Ann. Phys. 159, 220 (1985).

[26] Walter E. Thirring, A soluble relativistic field theory, Ann. Phys. 3, 91 (1958).

[27] Sidney Coleman, Quantum sine-Gordon equation as the massive Thirring model, Phys. Rev. D 11, 2088 (1975).

[28] David J. Gross and André Neveu, Dynamical symmetry breaking in asymptotically free field theories, Phys. Rev. D 10, 3235 (1974).

[29] R. Jackiw and C. Rebbi, Solitons with fermion number 1/2, Phys. Rev. D 13, 3398 (1976).

[30] M. C. Bañuls, R. Blatt, J. Catani, A. Celi, J. I. Cirac, M. Dalmonte, L. Fallani, K. Jansen, M. Lewenstein, S. Montangero, C. A. Muschik, B. Reznik, E. Rico, L. Tagliacozzo, K. Van Acoleyen, F. Verstraete, U. J. Wiese, M. Wingate, J. Zakrzewski, and P. Zoller, Simulating lattice gauge theories within quantum technologies, arXiv:1911.00003 (2019).

[31] Erez Zohar, J. Ignacio Cirac, and Benni Reznik, Quantum simulations of lattice gauge theories using ultracold atoms in optical lattices, Rep. Prog. Phys. 79, 014401 (2015).

[32] M. Dalmonte and S. Montangero, Lattice gauge theory simulations in the quantum information era, Contemp. Phys. 57, 388 (2016).

[33] U.-J. Wiese, Ultracold quantum gases and lattice systems: Quantum simulation of lattice gauge theories, Ann. Phys. 525, 777 (2013).

[34] Mari Carmen Bañuls and Krzysztof Cichy, Review on novel methods for lattice gauge theories, Rep. Prog. Phys. 83, 024401 (2020).

[35] Valentin Kasper, Gediminas Juzeliunas, Maciej Lewenstein, Fred Jendrzejewski, and Erez Zohar, From the Jaynes-Cummings model to non-Abelian gauge theories: A guided tour for the quantum engineer, arXiv:2006.01258 (2020).

[36] Jeff Greensite, Introduction to the Confinement Problem (Springer Nature, Cham, Switzerland, 2020).

[37] John B. Kogut and Mikhail A. Stephanov, The Phases of Quantum Chromodynamics: From Confinement to Extreme Environments, Cambridge Monographs on Particle Physics, Nuclear Physics and Cosmology (Cambridge University Press, Cambridge, UK, 2003). 
[38] A. Bazavov, H.-T. Ding, P. Hegde, O. Kaczmarek, F. Karsch, N. Karthik, E. Laermann, Anirban Lahiri, R. Larsen, S.-T. Li, Swagato Mukherjee, H. Ohno, P. Petreczky, H. Sandmeyer, C. Schmidt, S. Sharma, and P. Steinbrecher, Chiral crossover in QCD at zero and nonzero chemical potentials, Phys. Lett. B 795, 15 (2019).

[39] Han-Ning Dai, Bing Yang, Andreas Reingruber, Hui Sun, Xiao-Fan Xu, Yu-Ao Chen, Zhen-Sheng Yuan, and JianWei Pan, Four-body ring-exchange interactions and anyonic statistics within a minimal toric-code Hamiltonian, Nat. Phys. 13, 1195 (2017).

[40] Erez Zohar and Benni Reznik, Confinement and Lattice Quantum-Electrodynamic Electric Flux Tubes Simulated with Ultracold Atoms, Phys. Rev. Lett. 107, 275301 (2011).

[41] Erez Zohar, J. Ignacio Cirac, and Benni Reznik, Simulating Compact Quantum Electrodynamics with Ultracold Atoms: Probing Confinement and Nonperturbative Effects, Phys. Rev. Lett. 109, 125302 (2012).

[42] L. Tagliacozzo, A. Celi, A. Zamora, and M. Lewenstein, Optical abelian lattice gauge theories, Ann. Phys. 330, 160 (2013).

[43] L. Tagliacozzo, A. Celi, P. Orland, M. W. Mitchell, and M. Lewenstein, Simulation of non-abelian gauge theories with optical lattices, Nat. Commun. 4, 2615 (2013).

[44] G. K. Brennen, G. Pupillo, E. Rico, T. M. Stace, and D. Vodola, Loops and Strings in a Superconducting Lattice Gauge Simulator, Phys. Rev. Lett. 117, 240504 (2016).

[45] E. Rico, M. Dalmonte, P. Zoller, D. Banerjee, M. Bögli, P. Stebler, and U.-J. Wiese, $\mathrm{SO}(3)$ "nuclear physics" with ultracold gases, Ann. Phys. 393, 466 (2018).

[46] Jonghoon Park, Yoshihito Kuno, and Ikuo Ichinose, Glassy dynamics from quark confinement: Atomic quantum simulation of the gauge-Higgs model on a lattice, Phys. Rev. A 100, 013629 (2019).

[47] Fangli Liu, Rex Lundgren, Paraj Titum, Guido Pagano, Jiehang Zhang, Christopher Monroe, and Alexey V. Gorshkov, Confined Quasiparticle Dynamics in Long-Range Interacting Quantum Spin Chains, Phys. Rev. Lett. 122, 150601 (2019).

[48] W. L. Tan, P. Becker, F. Liu, G. Pagano, K. S. Collins, A. De, L. Feng, H. B. Kaplan, A. Kyprianidis, R. Lundgren, W. Morong, S. Whitsitt, A. V. Gorshkov, and C. Monroe, Observation of domain wall confinement and dynamics in a quantum simulator, arXiv:1912.11117 (2019).

[49] Umberto Borla, Ruben Verresen, Fabian Grusdt, and Sergej Moroz, Confined Phases of One-Dimensional Spinless Fermions Coupled to $Z_{2}$ Gauge Theory, Phys. Rev. Lett. 124, 120503 (2020).

[50] Simone Notarnicola, Mario Collura, and Simone Montangero, Real-time-dynamics quantum simulation of $(1+$ 1)-dimensional lattice QED with Rydberg atoms, Phys. Rev. Res. 2, 013288 (2020).

[51] Federica M. Surace, Paolo P. Mazza, Giuliano Giudici, Alessio Lerose, Andrea Gambassi, and Marcello Dalmonte, Lattice Gauge Theories and String Dynamics in Rydberg Atom Quantum Simulators, Phys. Rev. X 10, 021041 (2020).

[52] Giuseppe Magnifico, Marcello Dalmonte, Paolo Facchi, Saverio Pascazio, Francesco V. Pepe, and Elisa Ercolessi,
Real time dynamics and confinement in the $\mathbb{Z}_{n}$ SchwingerWeyl lattice model for 1+1 QED, Quantum 4, 281 (2020).

[53] Daniel González-Cuadra, Luca Tagliacozzo, Maciej Lewenstein, and Alejandro Bermudez, Robust Topological Order in Fermionic $\digamma_{2}$ Gauge Theories: From Aharonov-Bohm Instability to Soliton-Induced Deconfinement, Phys. Rev. X 10, 041007 (2020).

[54] Titas Chanda, Jakub Zakrzewski, Maciej Lewenstein, and Luca Tagliacozzo, Confinement and Lack of Thermalization After Quenches in the Bosonic Schwinger Model, Phys. Rev. Lett. 124, 180602 (2020).

[55] Fangli Liu, Seth Whitsitt, Przemyslaw Bienias, Rex Lundgren, and Alexey V. Gorshkov, Realizing and probing baryonic excitations in Rydberg atom arrays, arXiv:2007.07258 (2020).

[56] A. M. Polyakov, Interaction of Goldstone particles in two dimensions. Applications to ferromagnets and massive Yang-Mills fields, Phys. Lett. B 59, 79 (1975).

[57] Sidney Coleman, There are no Goldstone bosons in two dimensions, Commun. Math. Phys. 31, 259 (1973).

[58] F. D. M. Haldane, Continuum dynamics of the 1-d Heisenberg antiferromagnet: Identification with the o(3) nonlinear sigma model, Phys. Lett. A 93, 464 (1983).

[59] Ian Affleck, The quantum Hall effects, $\sigma$-models at $\Theta=$ $\pi$ and quantum spin chains, Nucl. Phys. B 257, 397 (1985).

[60] Subir Sachdev, Quantum Phase Transitions (Cambridge University Press, Cambridge, UK, 2011), 2nd ed.

[61] Jun Kondo, Resistance minimum in dilute magnetic alloys, Prog. Theor. Phys. 32, 37 (1964).

[62] D. Jaksch, C. Bruder, J. I. Cirac, C. W. Gardiner, and P. Zoller, Cold Bosonic Atoms in Optical Lattices, Phys. Rev. Lett. 81, 3108 (1998).

[63] W. Hofstetter, J. I. Cirac, P. Zoller, E. Demler, and M. D. Lukin, High-Temperature Superfluidity of Fermionic Atoms in Optical Lattices, Phys. Rev. Lett. 89, 220407 (2002).

[64] Alexander Albus, Fabrizio Illuminati, and Jens Eisert, Mixtures of bosonic and fermionic atoms in optical lattices, Phys. Rev. A 68, 023606 (2003).

[65] P. Jordan and E. Wigner, Über das paulische äquivalenzverbot, Z. Phys. 47, 631 (1928).

[66] F. D. M. Haldane, Luttinger liquid theory of onedimensional quantum fluids. I. Properties of the Luttinger model and their extension to the general 1d interacting spinless Fermi gas, J. Phys. C: Solid State Phys. 14, 2585 (1981).

[67] Sin Tomonaga, Remarks on Bloch's method of sound waves applied to many-fermion problems, Prog. Theor. Phys. 5, 544 (1950).

[68] J. M. Luttinger, An exactly soluble model of a manyfermion system, J. Math. Phys. 4, 1154 (1963).

[69] John Kogut and Leonard Susskind, Hamiltonian formulation of Wilson's lattice gauge theories, Phys. Rev. D 11, 395 (1975).

[70] Sidney Coleman, Aspects of Symmetry: Selected Erice Lectures (Cambridge University Press, Cambridge, UK, 1985).

[71] J. M. Radcliffe, Some properties of coherent spin states, J. Phys. A: Gen. Phys. 4, 313 (1971). 
[72] Eduardo Fradkin, Field Theories of Condensed Matter Physics (Cambridge University Press, Cambridge, UK, 2013), 2nd ed.

[73] R. E. Peierls, Quantum Theory of Solids, International Series of Monographs on Physics (Clarendon Press, 1955).

[74] Grabiele Giuliani and Giovani Vignale, Quantum Theory of the Electron Liquid (Cambridge University Press, Cambridge, UK, 2008).

[75] Ulrich Schollwöck, The density-matrix renormalization group in the age of matrix product states, Ann. Phys. 326, 96 (2011).

[76] Johannes Hauschild and Frank Pollmann, Efficient numerical simulations with tensor networks: Tensor network python (TeNPy), SciPost Phys. Lect. Notes 5, 1 (2018).

[77] Steven R. White, Density Matrix Formulation for Quantum Renormalization Groups, Phys. Rev. Lett. 69, 2863 (1992).

[78] L.-M. Duan, E. Demler, and M. D. Lukin, Controlling Spin Exchange Interactions of Ultracold Atoms in Optical Lattices, Phys. Rev. Lett. 91, 090402 (2003).

[79] S. Trotzky, P. Cheinet, S. Fölling, M. Feld, U. Schnorrberger, A. M. Rey, A. Polkovnikov, E. A. Demler, M. D. Lukin, and I. Bloch, Time-resolved observation and control of superexchange interactions with ultracold atoms in optical lattices, Science 319, 295 (2008).

[80] Daniel Greif, Thomas Uehlinger, Gregor Jotzu, Leticia Tarruell, and Tilman Esslinger, Short-range quantum magnetism of ultracold fermions in an optical lattice, Science 340, 1307 (2013).

[81] Martin Boll, Timon A. Hilker, Guillaume Salomon, Ahmed Omran, Jacopo Nespolo, Lode Pollet, Immanuel Bloch, and Christian Gross, Spin- and density-resolved microscopy of antiferromagnetic correlations in FermiHubbard chains, Science 353, 1257 (2016).

[82] Anton Mazurenko, Christie S. Chiu, Geoffrey Ji, Maxwell F. Parsons, Márton Kanász-Nagy, Richard Schmidt, Fabian Grusdt, Eugene Demler, Daniel Greif, and Markus Greiner, A cold-atom Fermi-Hubbard antiferromagnet, Nature 545, 462 (2017).

[83] Ivana Dimitrova, Niklas Jepsen, Anton Buyskikh, Araceli Venegas-Gomez, Jesse Amato-Grill, Andrew Daley, and Wolfgang Ketterle, Enhanced Superexchange in a Tilted Mott Insulator, Phys. Rev. Lett. 124, 043204 (2020).

[84] J. Sebby-Strabley, M. Anderlini, P. S. Jessen, and J. V. Porto, Lattice of double wells for manipulating pairs of cold atoms, Phys. Rev. A 73, 033605 (2006).

[85] S. Fölling, S. Trotzky, P. Cheinet, M. Feld, R. Saers, A. Widera, T. Müller, and I. Bloch, Direct observation of second-order atom tunnelling, Nature 448, 1029 (2007).

[86] S. Trotzky, Y-A. Chen, A. Flesch, I. P. McCulloch, U. Schollwöck, J. Eisert, and I. Bloch, Probing the relaxation towards equilibrium in an isolated strongly correlated one-dimensional Bose gas, Nat. Phys. 8, 325 (2012).

[87] Simon Fölling, Fabrice Gerbier, Artur Widera, Olaf Mandel, Tatjana Gericke, and Immanuel Bloch, Spatial quantum noise interferometry in expanding ultracold atom clouds, Nature 434, 481 (2005).
[88] T. Rom, Th. Best, D. van Oosten, U. Schneider, S. Fölling, B. Paredes, and I. Bloch, Free fermion antibunching in a degenerate atomic Fermi gas released from an optical lattice, Nature 444, 733 (2006).

[89] Stefan Trotzky, Yu-Ao Chen, Ute Schnorrberger, Patrick Cheinet, and Immanuel Bloch, Controlling and Detecting Spin Correlations of Ultracold Atoms in Optical Lattices, Phys. Rev. Lett. 105, 265303 (2010).

[90] W. S. Bakr, A. Peng, M. E. Tai, R. Ma, J. Simon, J. I. Gillen, S. Fölling, L. Pollet, and M. Greiner, Probing the superfluid-to-Mott insulator transition at the single-atom level, Science 329, 547 (2010).. American Association for the Advancement of Science Section: Report.

[91] Jacob F. Sherson, Christof Weitenberg, Manuel Endres, Marc Cheneau, Immanuel Bloch, and Stefan Kuhr, Singleatom-resolved fluorescence imaging of an atomic Mott insulator, Nature 467, 68 (2010).

[92] Joannis Koepsell, Sarah Hirthe, Dominik Bourgund, Pimonpan Sompet, Jayadev Vijayan, Guillaume Salomon, Christian Gross, and Immanuel Bloch, Robust Bilayer Charge Pumping for Spin- and Density-Resolved Quantum gas Microscopy, Phys. Rev. Lett. 125, 010403 (2020).

[93] Thomas Hartke, Botond Oreg, Ningyuan Jia, and Martin Zwierlein, Measuring total density correlations in a FermiHubbard gas via bilayer microscopy, arXiv:2003.11669 [cond-mat.quant-gas] (2020).

[94] Bing Yang, Hui Sun, Chun-Jiong Huang, Han-Yi Wang, Youjin Deng, Han-Ning Dai, Zhen-Sheng Yuan, and JianWei Pan, Cooling and entangling ultracold atoms in optical lattices, Science 369, 550 (2020).

[95] Sidney Coleman and Edward Witten, Chiral-Symmetry Breakdown in Large- $n$ Chromodynamics, Phys. Rev. Lett. 45, 100 (1980).

[96] Frank Wilczek, Mass without mass I: Most of matter, Phys. Today 52, 11 (1999).

[97] R. Rapp and J. Wambach, in Advances in Nuclear Physics, edited by J. W. Negele and Erich Vogt (Springer, USA, Boston, MA, 2000), p. 1.

[98] Y. Nambu and G. Jona-Lasinio, Dynamical model of elementary particles based on an analogy with superconductivity. I, Phys. Rev. 122, 345 (1961).

[99] N. Brambilla, QCD and strongly coupled gauge theories: Challenges and perspectives, Eur. Phys. J. C 74, 2981 (2014).

[100] Larry McLerran and Robert D. Pisarski, Phases of dense quarks at large nc, Nucl. Phys. A 796, 83 (2007).

[101] W. P. Su, J. R. Schrieffer, and A. J. Heeger, Solitons in Polyacetylene, Phys. Rev. Lett. 42, 1698 (1979).

[102] Gunnar S. Bali, QCD forces and heavy quark bound states, Phys. Rep. 343, 1 (2001).

[103] Sidney Coleman, R. Jackiw, and Leonard Susskind, Charge shielding and quark confinement in the massive Schwinger model, Ann. Phys. 93, 267 (1975).

[104] Boye Buyens, Jutho Haegeman, Henri Verschelde, Frank Verstraete, and Karel Van Acoleyen, Confinement and String Breaking for $\mathrm{QED}_{2}$ in the Hamiltonian Picture, Phys. Rev. X 6, 041040 (2016).

[105] A. Chodos, R. L. Jaffe, K. Johnson, C. B. Thorn, and V. F. Weisskopf, New extended model of hadrons, Phys. Rev. D 9, 3471 (1974). 
[106] W. A. Bardeen, M. S. Chanowitz, S. D. Drell, M. Weinstein, and T. M. Yan, Heavy quarks and strong binding: A field theory of hadron structure, Phys. Rev. D 11, 1094 (1975).

[107] R. Friedberg and T. D. Lee, Fermion-field nontopological solitons, Phys. Rev. D 15, 1694 (1977).

[108] R. Friedberg and T. D. Lee, Fermion-field nontopological solitons. II. Models for hadrons, Phys. Rev. D 16, 1096 (1977).

[109] D. K. Campbell and A. R. Bishop, Soliton excitations in polyacetylene and relativistic field theory models, Nucl. Phys. B 200, 297 (1982).

[110] Daniel González-Cuadra, Alexandre Dauphin, Przemysław R. Grzybowski, Paweł Wójcik, Maciej Lewenstein, and Alejandro Bermudez, Symmetry-breaking topological insulators in the $\digamma_{2}$ Bose-Hubbard model, Phys. Rev. B 99, 045139 (2019).

[111] Daniel González-Cuadra, Alejandro Bermudez, Przemysław R. Grzybowski, Maciej Lewenstein, and Alexandre Dauphin, Intertwined topological phases induced by emergent symmetry protection, Nat. Commun. 10, 2694 (2019).

[112] Daniel González-Cuadra, Alexandre Dauphin, Przemysław R. Grzybowski, Maciej Lewenstein, and Alejandro Bermudez, $\mathbb{Z}_{n}$ solitons in intertwined topological phases, arXiv:1908.02186 (2019).

[113] Daniel González-Cuadra, Alexandre Dauphin, Przemysław R. Grzybowski, Maciej Lewenstein, and Alejandro Bermudez, Dynamical solitons and boson fractionalization in cold-atom topological insulators, arXiv:2003. 10994 (2020).

[114] A. Bermudez, E. Tirrito, M. Rizzi, M. Lewenstein, and S. Hands, Gross-Neveu-Wilson model and correlated symmetry-protected topological phases, Ann. Phys. 399, 149 (2018).

[115] E. Tirrito, M. Rizzi, G. Sierra, M. Lewenstein, and A. Bermudez, Renormalization group flows for WilsonHubbard matter and the topological Hamiltonian, Phys. Rev. B 99, 125106 (2019).

[116] J. Jünemann, A. Piga, S.-J. Ran, M. Lewenstein, M. Rizzi, and A. Bermudez, Exploring Interacting Topological Insulators with Ultracold Atoms: The Synthetic Creutz-Hubbard Model, Phys. Rev. X 7, 031057 (2017).

[117] G. Magnifico, D. Vodola, E. Ercolessi, S. P. Kumar, M. Müller, and A. Bermudez, Symmetry-protected topological phases in lattice gauge theories: Topological qed $_{2}$, Phys. Rev. D 99, 014503 (2019).

[118] G. Magnifico, D. Vodola, E. Ercolessi, S. P. Kumar, M. Müller, and A. Bermudez, $\digamma_{N}$ gauge theories coupled to topological fermions: $\mathrm{QED}_{2}$ with a quantum mechanical $\theta$ angle, Phys. Rev. B 100, 115152 (2019).

[119] Andrea Simoni, Matteo Zaccanti, Chiara D'Errico, Marco Fattori, Giacomo Roati, Massimo Inguscio, and Giovanni Modugno, Near-threshold model for ultracold KRb dimers from interisotope Feshbach spectroscopy, Phys. Rev. A 77, 052705 (2008).

[120] Jacob Johansen, B. J. DeSalvo, Krutik Patel, and Cheng Chin, Testing universality of Efimov physics across broad and narrow Feshbach resonances, Nat. Phys. 13, 731 (2017).

[121] N. Kawamoto and J. Smit, Effective lagrangian and dynamical symmetry breaking in strongly coupled lattice QCD, Nucl. Phys. B 192, 100 (1981). 